No $2010-21$

Octobre 2010

Réforme des retraites en France : évaluation de la mise en place d'un système par comptes notionnels

Xavier Chojnicki et Riccardo Magnani 


\section{TABLE DES MATIÈRES}

Résumé non technique . . . . . . . . . . . . . . . . . . . . . . . . . . . 3

Résumé court . . . . . . . . . . . . . . . . . . . . . . . . . . . . . . 4

Non-technical summary. . . . . . . . . . . . . . . . . . . . . . 5

Abstract . . . . . . . . . . . . . . . . . . . . . . . . . . . . . . . 6

1. Introduction . . . . . . . . . . . . . . . . . . . . . . . . . . . . . . 7

2. Population et activité . . . . . . . . . . . . . . . . . . . . . . 10

2.1. L'évolution démographique . . . . . . . . . . . . . . . . . . . . . . 10

2.2. La population active et sa structure . . . . . . . . . . . . . . . . . . . 13

2.3. Les retraités . . . . . . . . . . . . . . . . . . . . . . . . . . . . 14

3. La structure du modèle. . . . . . . . . . . . . . . . . . . . . . . . . . 15

3.1. Le secteur productif . . . . . . . . . . . . . . . . . . . . . . . . . 16

3.2. Salaire et chômage d'équilibre . . . . . . . . . . . . . . . . . . . . . 17

3.3. Les comportements individuels. . . . . . . . . . . . . . . . . . . . . 17

4. Les pensions de retraite publiques . . . . . . . . . . . . . . . . . . . . 19

4.1. Le régime des fonctionnaires $\left(" \mathrm{rf}^{\prime \prime}\right)$. . . . . . . . . . . . . . . . . . . 19

4.2. Le régime de base $(" r b ")$. . . . . . . . . . . . . . . . . . . . . . . 20

4.3. Les régimes complémentaires ("rc") . . . . . . . . . . . . . . . . . . . 21

5. Dynamique et bouclage macroéconomique . . . . . . . . . . . . . . . . . . 23

5.1. Equilibre des caisses de retraite. . . . . . . . . . . . . . . . . . . . 23

5.2. Equilibre du marché financier . . . . . . . . . . . . . . . . . . . . . 25

6. Evaluation à moyen terme de l'évolution du système de retraite. . . . . . . . . . 26

6.1. Le scénario de référence . . . . . . . . . . . . . . . . . . . . . . . 26

6.2. Introduction d'un système par comptes notionnels . . . . . . . . . . . . . 30

7. Evaluation de la stabilité du système par comptes notionnels . . . . . . . . . . . 35

7.1. Comptes notionnels et chocs démographiques . . . . . . . . . . . . . . 36

7.2. Comptes notionnels et chocs économiques . . . . . . . . . . . . . . . . 37

7.3. Comptes notionnels et chocs institutionnels . . . . . . . . . . . . . . . . 39

8. Conclusion . . . . . . . . . . . . . . . . . . . . . . 40

Références . . . . . . . . . . . . . . . . . . . . . . . . . 42

Liste des papiers du CEPII. . . . . . . . . . . . . . . . . . . . . . . . . . 44 


\section{RÉFORME DES RETRAITES EN FRANCE : ÉVALUATION DE LA MISE EN PLACE D'UN SYSTÈME PAR COMPTES NOTIONNELS}

\section{RÉSUME NON TECHNIQUE}

Malgré plusieurs réformes d'importance depuis le milieu des années 1990, la pérennité financière du système français de retraite n'est pas assurée. Ce système, qui est basé sur le principe de la répartition, n'a pas été conçu pour absorber un choc démographique comme celui que va connaître la population française à l'horizon du demi-siècle à venir. En effet, l'allongement continu de la durée de vie induit, en l'absence de réformes, un accroissement de la période de retraite qui pèse sur l'équilibre du système. Le départ massif à la retraite des générations du baby-boom entre 2005 et 2030 diminue, en outre, fortement le nombre de cotisants par retraité.

Après plusieurs réformes paramétriques est aujourd'hui envisagée une réforme structurelle du système, conservant le principe de la répartition, mais réorganisant celle-ci pour passer à un système par comptes notionnels. Dans ce système, les pensions de chaque individu sont calculées de façon à égaliser la valeur des cotisations qu'il a versées et la valeur des pensions qu'il va recevoir au cours de sa période de retraite. Une telle réforme pourrait être l'occasion de remplacer un système où coexistent une multitude de caisses aux règles de fonctionnement différentes par un système plus homogène dont la gestion serait plus simple et transparente. Elle permettrait aussi de prendre en compte automatiquement, dans le calcul des pensions, tout accroissement de l'espérance de vie et toute modification du rythme de croissance économique.

Nous évaluons les effets d'une transformation du système actuel de retraite français en un système par comptes notionnels. L'analyse est effectuée à l'aide du modèle OLGAMAP, développé au CEPII. Il s'agit d'un modèle d'équilibre général à générations imbriquées qui, par rapport à la plupart des modèles utilisés dans ce type d'analyse, a l'avantage de prendre en compte les effets de bouclage macroéconomique sous différentes hypothèses d'ouverture économique. Ainsi, les effets de retour des réformes des retraites sur les marchés du capital et du travail ainsi que sur la croissance sont calculés par le modèle et non définis de manière exogène, ce qui affecte finalement l'effet des réformes sur l'équilibre des régimes des retraites.

En particulier, nous simulons les effets de la mise en place progressive, entre 2015 et 2030, d'un système unique par comptes notionnels se substituant, à terme, entièrement à tous les régimes actuels, de base et complémentaires. L'introduction d'un système de comptes notionnels nécessite plusieurs choix de la part du décideur politique. En particulier, nous considérons ici un taux de cotisation fixé de manière à maintenir constante la masse des cotisations découlant de l'ancien système à la date de début de la transition; un taux de rendement appliqué aux cotisations versées égal au taux de croissance annuel du PIB ; un taux d'actualisation des pensions égal au taux de croissance de la productivité; enfin, une indexation des pensions sur les prix. Par rapport à notre scénario de référence, les résultats des simulations montrent que l'introduction progressive d'un système par comptes notionnels permettrait d'obtenir une réduction des besoins de financement de l'ordre de 0,6 point de PIB à l'horizon 2050. 
Nous évaluons ensuite la stabilité du système par comptes notionnels face à un certain nombre de chocs démographiques, économiques et institutionnels. Pour chacun des chocs considérés, nous étudions la réaction des deux systèmes de retraite (le système actuel et celui par comptes notionnels). En termes de besoins de financement, les résultats de nos simulations montrent que le système par comptes notionnels réagit mieux, par rapport au système actuel, dans le cas d'une augmentation de l'espérance de vie, d'un ralentissement de la productivité et d'une baisse des taux d'activité. Le nouveau système réagit de façon très similaire dans le cas d'une baisse de la fécondité et d'une hausse du taux de chômage. Par contre, une hausse du taux de cotisations dégrade l'équilibre d'un système par comptes notionnels du fait d'un accroissement parallèle de la valeur des pensions, lié au caractère purement contributif du nouveau système.

\section{RÉSUMÉ COURT}

L'objectif est d'évaluer les effets d'une transformation du système actuel de retraite français en un système par comptes notionnels. L'analyse est effectuée à l'aide d'un modèle d'équilibre général à générations imbriquées qui permet de prendre en compte les effets de bouclage macroéconomique sous différentes hypothèses d'ouverture économique. Nous simulons les effets de la mise en place progressive, entre 2015 et 2030, d'un système unique par comptes notionnels se substituant, à terme, entièrement à tous les régimes actuels, de base et complémentaires. Par rapport à notre scénario de référence, les simulations montrent que l'introduction progressive d'un système par comptes notionnels permettrait d'obtenir une réduction des besoins de financement de l'ordre de 0,6 point de PIB à l'horizon 2050. Ensuite, nous évaluons la stabilité du système par comptes notionnels. Si le système par comptes notionnels réagit mieux que le système actuel dans les cas d'une augmentation de l'espérance de vie, d'un ralentissement de la productivité et d'une baisse des taux d'activité, il réagit de façon très similaire dans le cas d'une baisse de la fécondité et d'une hausse du taux de chômage. Par contre, une hausse des cotisations a un effet négatif sur les besoins de financement dans un système par comptes notionnels du fait d'un accroissement parallèle de la valeur des pensions.

Classification JEL : H55, J1, C68

Mots clés : $\quad$ Retraites; Vieillissement; Modèle OLG 


\section{REFORMING THE FRENCH PENSION SYSTEM: TRANSITION TO A NOTIONAL ACCOUNT}

SYSTEM

\section{NON-TECHNICAL SUMMARY}

The French pension system is based on a PAYG financing: today's contributors finance the pensions of today's pensioners and at the same time acquire rights to a pension that will be financed by tomorrow's contributors. It was not designed to deal with a demographic shock such as the one expected for the French population in the next fifty years. Without any new reforms, the continual extension of life expectancy will lead to an increase in the retirement length that will affect the system's balance. In addition, the mass retirement of the baby-boom generations between 2005 and 2030 will significantly reduce the number of contributors for each pensioner, even though, unlike its neighbours, France can rely on a fertility rate close to the generation renewal rate.

After several parametrical reforms, a transition to a notional-account system is now debated. In this system, which has already been introduced in Sweden and Italy, each individual's pension is calculated so that the value of all the pensions that he or she will receive during retirement is equal to the value of all the contributions that he or she has paid. Therefore, the basic principle is modeled on capitalisation, since contributions paid each year are virtually credited to an individual account. However, the system remains a PAYG one, which protects it from the uncertainties related to the evolution of the financial markets rate of return. Such a reform could also be the opportunity to transform a system, in which a multitude of funds with different operating rules coexist, in a more uniform one, both simpler and more transparent. It would also be able to automatically take into account any increase in life expectancy or change in the rate of economic growth in the calculation of pensions.

The purpose of this paper is to evaluate the consequences of the transition of the French current pension system to a notional-accounts pension system. We use the OLGAMAP model developed by the CEPII which is a stylized overlapping-generations general equilibrium model. The main advantage is to take into account macroeconomic feedback effects. Thus the reforms' feedback effects on capital and labour markets as well as on economic growth are computed within the model and not defined exogenously, which will finally affect the reforms' impact on the financial situation of the pension system.

In particular, in this paper, we have simulated the effects of a gradual setting up of a notional-account system between 2015 and 2030, replacing all of the current schemes (basic and complementary) in the long term. Policy-makers have to make several choices if they introduce a notional accounts system. Here, we assume that the contribution rate is set so that the mass of contributions coming from the old system at the start date of transition is constant, the rate of return on contributions is fixed at the payroll growth rate, the pensions discount rate is set at the productivity growth rate and the pensions are indexed on prices (and therefore remain constant in real terms). The simulation results show that gradually introducing a notional accounts system would reduce the financial needs by around 0.6 points of GDP in 2050. 
Then, we study the stability of the notional accounts system to demographic, economic and institutional shocks compared to the current system. In term of financial needs, the results of the notional accounts system are better than those of the current one in case of an increase in life expectancy, a decrease in the productivity growth rate and a decrease in the activity rate. The two systems are equivalent in the case of a decrease in fertility and an increase in unemployment rate. However, in the case of an increase in the contribution rate, the effect on the financial needs in a notional accounts system is negative, given that pensions mechanically increase in line with contributions.

\section{Abstract}

The purpose of this paper is to evaluate the consequences of the transition of the French current pension system to a notional-account pension system. We use the OLGAMAP model developed by the CEPII which is a stylised overlapping-generations general equilibrium model. The main advantage is to take into account the macroeconomic feedback effects. We simulate the effects of a gradual setting up of a global notional accounts system between 2015 and 2030, completely replacing all of the current schemes (basic and complementary) in the long term. The simulation results show that gradually introducing a notional-accounts system would reduce the financial needs by around 0.6 points of GDP in 2050. Then, we study the stability of the system. In term of financial needs, the results of the notional-accounts system are better than those of the current one in the case of an increase in life expectancy, a decrease in the productivity growth rate and a decrease in activity rate. The two systems are equivalent in the case of a decrease in fertility and an increase in the unemployment rate. However, in the case of an increase in the contribution rate, the effect on the financial needs in a notional-accounts system is negative, given that pensions mechanically increase in line with contributions.

\section{JEL Classification: $\mathrm{H} 55, \mathrm{~J} 1, \mathrm{C} 68$}

Keywords: $\quad$ Pension systems; Ageing; OLG model 


\title{
RÉFORME DES RETRAITES EN FRANCE : ÉVALUATION DE LA MISE EN PLACE D’UN SYSTÈME PAR COMPTES NOTIONNELS ${ }^{1}$
}

\author{
Xavier Chojnicki ${ }^{*}$, Riccardo Magnani ${ }^{\dagger}$
}

\section{INTRODUCTION}

Malgré plusieurs réformes d'importance depuis le milieu des années 1990, la pérennité financière du système français de retraite n'est pas assurée et soulève de nombreuses inquiétudes quant à l'évolution du niveau des retraites. Ce système est basé sur le principe de la répartition : les cotisants d'aujourd'hui financent les pensions des retraités d'aujourd'hui et s'ouvrent en parallèle des droits à la retraite qui seront financés par les cotisants de demain. Ce système ne peut fonctionner correctement qu'en régime démographique stable ou d'expansion démoéconomique comme durant les Trente Glorieuses. Il n'a pas été conçu pour absorber un choc démographique comme celui que va connaître la population française à l'horizon du demi-siècle à venir. En effet, l'allongement continu de la durée de vie induit, en l'absence de réformes, un accroissement de la période de retraite qui pèse sur l'équilibre du système. Le départ massif à la retraite des générations du baby-boom entre 2005 et 2030 diminue, en outre, fortement le rapport du nombre de cotisants par retraité, même si, contrairement à ses voisins, la France peut compter sur une fécondité proche du seuil de renouvellement des générations.

Après plusieurs réformes paramétriques (la dernière en date relevant notamment l'âge légal de départ à la retraite), est aujourd'hui évoquée une réforme structurelle du système, conservant le principe de la répartition mais en la réorganisant pour passer à un système par comptes notionnels. Dans ce système, déjà introduit en Suède et en Italie, les pensions de chaque individu sont calculées de façon à égaliser la valeur des cotisations qu'il a versées et la valeur des pensions qu'il va recevoir au cours de sa période de retraite. Le principe de base est donc inspiré de la capitalisation, les cotisations acquittées chaque année étant virtuellement créditées sur un compte individuel. Toutefois, le système continue de fonctionner par répartition, ce qui le préserve des incertitudes sur le rendement des marchés financiers. Une telle réforme pourrait également être l'occasion de rendre plus homogène un système où coexistent une multitude de caisses aux règles de fonctionnement différentes, en lui substituant un système dont la gestion serait plus simple et transparente. Elle permettrait aussi de prendre en compte automatiquement, dans le calcul des pensions, tout accroissement de l'espérance de vie et toute modification du rythme de croissance économique.

1. Correspondance : xavier.chojnicki@univ-lille2.fr

*. EQUIPPE, Université de Lille 2 et CEPII.

$\dagger$. CEPN, Université de Paris 13 et CEPII. 
Le passage à un système par comptes notionnels a été défendu récemment par Bozio \& Piketty (2008). Il a également été étudié à la demande du parlement par le Conseil d'Orientation des Retraites (2010b). Dans son septième rapport, le COR présente les modalités techniques et juridiques de la transition vers un tel système, ainsi que plusieurs évaluations réalisées par son Secrétariat général (sur la base d'un modèle stylisé), par l'INSEE (en supposant une application immédiate du nouveau système) et par la Caisse Nationale d'Assurance Vieillesse (uniquement pour le régime général). Aucun de ces travaux ne permet cependant d'analyser, dans un cadre d'équilibre général, les effets en termes de financement des caisses de retraites, de redistribution intergénérationnelle et de transition institutionnelle. Dans cet objectif, nous utilisons une version actualisée du modèle OLGAMAP (OverLapping Generations Applied Model to Ageing and Pensions $)^{2}$ développé au CEPII dont le principal avantage est de prendre en compte les effets de bouclage macroéconomique pour différents environnements d'ouverture économique. Ainsi, les effets de retour des réformes sur les marchés du capital et du travail ainsi que sur la croissance sont calculés par le modèle et non définis de manière exogène, ce qui affecte finalement l'effet des réformes sur l'équilibre des régimes des retraites.

Plusieurs outils de prospective quantitative ont été élaborés ces dernières années dans la majorité des pays développés pour répondre aux questions soulevées par l'évolution du système de retraite en réaction au vieillissement démographique. Une première distinction de fond peut alors être proposée entre les modèles d'équilibre général, d'une part, et les modèles "comptables", d'autre part, dans lesquels la plupart des grandeurs macro-économiques sont exogènes, ce qui revient à effectuer des analyses d'équilibre partiel. Une autre source de distinction porte sur la nature, plus ou moins précise, des fondements théoriques à l'origine des comportements économiques des agents du modèle.

Les modèles d'équilibre partiel utilisent des scénario démographiques et économiques exogènes (activité, emploi, productivité et coût des facteurs). Les modèles agrégés d'optique comptable, telle la maquette de projection du système de retraite réalisée à la Drees (Bac, Bonnet, Bontout \& Cornilleau (2003)) ou encore les projections effectuées par le COR ((2001, 2006, 2007, 2010a)), raisonnent implicitement avec un agent représentatif (par génération et par genre) et sous une hypothèse de stabilité du ratio du capital par unité de travail efficace. Autrement dit, l'économie se situe implicitement sur son sentier de croissance équilibrée de long terme. La finalité de ces instruments est essentiellement d'analyser l'impact de variantes sociodémographiques et de réformes paramétriques des régimes de retraite sur la soutenabilité de ceux-ci. Les modèles de micro-simulations tels celui de l'équipe Destinie (Bardaji, Sédillot \& Walraet (2004)) intègrent des scénarios macro-économiques exogènes, notamment en matière de production et de prix des facteurs. Leur objectif est d'analyser l'ensemble des trajectoires

2. Par rapport à la précédente version du modèle Olgamap (Chojnicki \& Magnani (2008) et Chateau, Chojnicki \& Magnani (2009)), la version ici utilisée se distingue par un (1) important travail d'actualisation, notamment pour intégrer les effets de la crise récente, (2) le développement d'un module spécifique permettant de différencier l'âge de départ à la retraite à l'intérieur d'une génération ainsi que (3) l'introduction d'un rendement différencié entre le capital physique et les titres sur la dette publique. 
individuelles d'une multitude d'agents face à des changements des prélèvements et transferts sociaux et budgétaires. L'accent est donc mis sur les aspects redistributifs des réformes sans que ceux-ci interagissent avec les variables macro-économiques. Le principal inconvénients de ces modèles est de ne pas tenir compte des effets du bouclage macroéconomique.

La seconde catégorie d'outils est constituée par des modèles d'équilibre général. Les modèles micro-fondés appliqués aux questions liées au vieillissement démographique se sont développés à la suite du modèle d'équilibre général calculable d'Auerbach \& Kotlikoff (1987) qui s'appuie sur le cadre théorique du modèle à générations imbriquées d'agents (Samuelson (1958), Diamond (1965)). Alimenté par des projections démographiques exogènes, la dynamique de l'économie est alors entièrement expliquée par les réactions des agents rationnels et optimisateurs, et en particulier par leur comportement d'épargne pour motif de cycle de vie dans un cadre d'anticipations parfaites. Les analyses effectuées grâce à ce type de modèle souffrent d'un défaut majeur : la complexité de ces modèles interdit en général de tenir compte d'une hétérogénéité des individus au sein d'une même cohorte et empêchent donc de mener des études similaires à celles effectuées grâce à des modèles de micro-simulations.

Certains travaux récents, de nature plus académique, cherchent cependant à rendre compte de façon conjointe de l'hétérogénéité des trajectoires individuelles, de la distribution des richesses et des grandeurs des variables agrégées (Hairault, Langot \& Sopraseuth (2004), Hénin \& Weitzenblum (2004)). Cependant l'extrême complexité du calcul numérique des dynamiques endogènes de l'économie incite en général à simplifier à l'extrême certains comportements et mécanismes macroéconomiques clés. Ces travaux perdent alors de leur vertu en termes de prospective quantitative.

Afin d'aborder de façon plus spécifique des considérations macro-économiques, et non pas seulement comptables, mais aussi distributives en matière de financement des régimes de retraite, nous développons ici un modèle "hybride" (OLGAMAP). Il s'agit d'un modèle d'équilibre général dans lequel seuls les comportements clés en matière de réaction au phénomène du vieillissement démographique sont spécifiés de façon détaillée (la rémunération des facteurs, l'accumulation de la richesse par âge, l'évolution des revenus au cours du cycle de vie, ...). En revanche, d'autres aspects macro-économiques et comportementaux n'ont pas réellement besoin d'être mis en avant et sont, de ce fait, soit considérés comme exogènes (lorsque le lien entre leur évolution et la déformation de la pyramide des âges reste ténu comme c'est le cas pour les comportements individuels d'activité), soit schématisés (lorsque l'évolution de ces variables joue un rôle non négligeable sur la dynamique globale de l'économie mais sans pour autant que le lien avec le vieillissement soit direct, telle l'évolution du taux de chômage agrégé).

Le modèle OLGAMAP est un modèle d'équilibre général à générations d'agents hétérogènes. Ce modèle de croissance est dans l'esprit de celui proposé par Blanchet (1992) : les anticipations des agents restent statiques et les propensions marginales à consommer pour chaque groupe d'âge sont fixées. Les comportements d'épargne et la spécification du marché du travail sont comparables à ceux proposés dans le modèle exposé par d'Autume \& Quinet (2001). La prise 
en compte d'agents hétérogènes au sein d'une même cohorte permet, en outre, d'approfondir l'analyse menée avec les modèles à générations traditionnels notamment en évaluant l'impact des règles propres au système de retraite français sur la situation des agents.

Nous analysons ici les effets d'une transformation du système actuel de retraite français en un système par comptes notionnels. Nous simulons un premier scénario avec mise en place progressive, entre 2015 et 2030, d'un système unique par comptes notionnels se substituant entièrement, à terme, à tous les régimes actuels, de base et complémentaires. Nous montrons que des effets positifs en termes de financement peuvent apparaître avec la mise en place d'un système par comptes notionnels mais les gains financiers réalisés à l'horizon des prochaines décennies sont entièrement dépendants du paramétrage du système. De plus, des effets redistributifs importants peuvent également découler de la transformation de notre système de retraite actuel, où coexistent une multitude de régimes de retraite aux règles de fonctionnement différentes, en un régime unique. Cependant, les évaluations ici présentées reposent sur des hypothèses incertaines (démographie, progrès technique, etc.). Face à ces incertitudes, nous montrons qu'un système de comptes notionnels présente l'avantage d'une meilleure adaptabilité aux évolutions observées (en particulier s'agissant de l'évolution de l'espérance de vie et du rythme de la productivité).

La suite de cet article se présente de la manière suivante. Dans la section 2, nous exposons les hypothèses effectuées en matière de démographie et de participation au marché du travail. Puis la section 3 présente le cadre macroéconomique d'analyse et la section 4 la modélisation du système de retraite actuellement en place. Nous présentons ensuite l'équilibre du modèle (section 5) puis discutons des effets de la mise en place d'un système par comptes notionnels (section 6). Enfin, la section 7 compare les mécanismes d'ajustement d'un système par comptes notionnels par rapport au système actuel suite à des chocs démographiques (sur la fécondité et sur la durée de vie), économiques (sur la productivité, le taux de chômage et sur les taux d'activité) et sur les paramètres institutionnels (taux de cotisation et âge légal de départ à la retraite).

\section{Population et activité}

A chaque période co-existent différentes générations $g$, chacune se trouvant dans une phase différente de son cycle de vie. Les individus se distinguent, en outre, par leur genre $s$ et leur catégorie professionnelle $c$. L'horizon temporel de l'étude est la période 2005-2050, les générations concernées par le modèle sont donc celles nées entre 1899 et 2049 puisqu'à chaque date la pyramide des âges décrit la répartition des individus âgés de 0 à 105 ans. L'âge de chaque individu est défini simplement à la date $t$ par $a(t, g)=t-g$.

\subsection{L'évolution démographique}

Les interrogations sur les effets économiques du vieillissement démographique en France ont sensiblement évolué depuis le début des années 2000, en raison de la nouvelle donne démogra- 
phique. Contrairement aux projections précédentes de l'INSEE (Brutel \& Omalek (2003)), le processus de vieillissement démographique ne s'accompagne plus d'un déclin démographique. Les nouvelles perspectives démographiques positionnent la France dans une situation plus favorable que ses principaux partenaires de l'Union Européenne. Cette situation singulière résulte de la conjonction de deux changements récents : le taux de fécondité se maintient à un niveau plus élevé depuis le début de l'année 2000, sur la même période le solde migratoire a été sensiblement revu à la hausse (celui-ci a été en moyenne multiplié par deux par rapport aux années 1990). Le dernier scénario central des projections de l'INSEE (Robert-Bobée (2006)) intègre ces modifications en retenant un indice conjoncturel de fécondité qui se maintient à 1,9 enfant par femme (contre 1,8 enfant par femme dans les projections antérieures) et un solde migratoire net annuel identique à celui constaté pour les années 2004-2005, à savoir +100000 personnes par an (soit précisément le double de celui retenu dans les projections précédentes). Une autre évolution doit être signalée, car elle accentue l'évolution favorable pour le financement des régimes de retraite : sur la base de l'évolution constatée lors des 15 dernières années, et non plus les 30 dernières, le rythme de baisse de la mortalité est plus modéré, ce qui conduit à l'horizon 2050 à une espérance de vie à la naissance (Figure 2) pour les hommes de 83,8 ans (contre 84,3 dans les précédentes projections) et pour les femmes de 89 ans (contre 91 ans).

Ces nouvelles hypothèses démographiques sont introduites dans le bloc démographique du modèle, qui reproduit donc parfaitement le scénario central de l'INSEE. Avant 2005, les pyramides des âges utilisées proviennent des données historiques. Ce nouveau visage de la population française à l'horizon du demi-siècle à venir est marqué par les traits suivants :

- La population totale de la France en 2050 devrait s'élever à environ 70 millions d'habitants, soit un gain de plus de 9 millions d'individus par rapport à 2005 (tableau 1).

- Ces perspectives favorables se retrouvent dans l'évolution de la population en âge de travailler. Elle connaît une hausse sur la décennie qui vient de se terminer pour atteindre 40,5 millions d'individus en 2010 avant de s'inscrire sur une trajectoire légèrement à la baisse jusqu'en 2040 avec quasiment 40 millions d'individus à cette date. La baisse, relativement modérée, entre 2010 et 2040 (-0,8\%), est sans commune mesure avec celle qui était anticipée dans les projections démographiques précédentes de l'Insee (elle devait atteindre $-8,2 \%$ en 2040).

- Si l'on retient l'image traditionnelle de " l'effet de ciseaux " (augmentation sensible du nombre de personnes âgées et réduction de la population en âge de travailler) pour caractériser les perspectives démographiques, les nouvelles projections ne conservent que "l'effet de levier" de la longévité. Cependant, les données du tableau 1 mettent en évidence que c'est bel et bien cet allongement de l'espérance de vie qui est la cause principale du vieillissement démographique. Le ratio de dépendance est un bon indicateur de ce processus. Il devrait passer de 25,2\% en 2005 à plus de $45 \%$ en 2050.

Ce processus de vieillissement de la population française peut s'inscrire dans une perspective historique plus longue. Sur un horizon d'un siècle, la structure la population va ainsi connaître une déformation importante comme le montre la figure 1. Le vieillissement accroît ainsi progressivement le poids des générations les plus âgées : en 2005 (et encore plus en 1950), les 
TABLEAU 1 - Perspectives d'évolution de la population française

\begin{tabular}{lrrrrrr}
\hline \hline & $\mathbf{2 0 0 5}$ & $\mathbf{2 0 1 0}$ & $\mathbf{2 0 2 0}$ & $\mathbf{2 0 3 0}$ & $\mathbf{2 0 4 0}$ & $\mathbf{2 0 5 0}$ \\
\hline Population totale (en milliers) & 60702 & 62302 & 64984 & 67204 & 69019 & 69961 \\
\hline Population en âge de travailler (15-64 ans, en milliers) & 39531 & 40532 & 40499 & 40333 & 39984 & 40224 \\
\hline Population de plus de 65 ans & 9968 & 10377 & 13080 & 15620 & 17656 & 18323 \\
\hline Ratio de dépendance (Pop 65 et plus / Pop 15-64 en \%) & $25,2 \%$ & $25,6 \%$ & $32,3 \%$ & $38,7 \%$ & $44,2 \%$ & $45,6 \%$ \\
\hline Nombre de cotisants (en milliers) & 25096 & 25842 & 26212 & 26255 & 26429 & 26412 \\
\hline Nombre de retraités (en milliers) & 13225 & 14962 & 17863 & 20535 & 22247 & 23109 \\
\hline Nombre de cotisants par retraité & 1,90 & 1,73 & 1,47 & 1,28 & 1,19 & 1,14 \\
\hline \hline
\end{tabular}

Insee (2006), Calculs des auteurs.

classes d'âge les plus nombreuses appartiennent aux catégories en âge d'activité alors qu'en 2050, les deux premières générations de retraités (soixantenaires et septuagénaires) sont aussi nombreuses que les générations en âge d'activité. Les générations les plus âgées augmentent en même temps très fortement, les plus de 90 ans par exemple étant multipliés quasiment par quatre entre 2005 et 2050 .

FigURE 1 - Population par grands groupes d'âge en 1950, 2005 et 2050

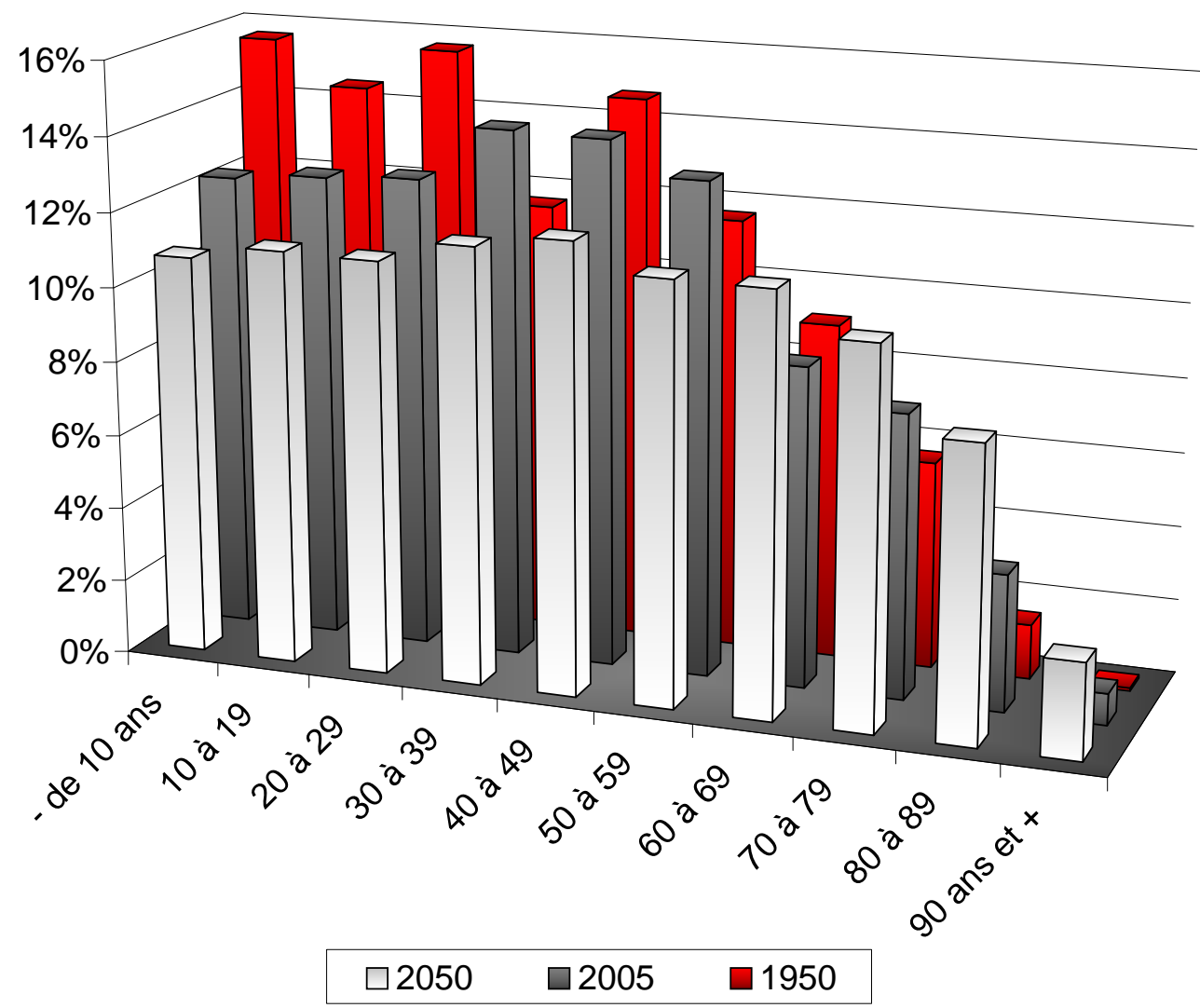




\subsection{La population active et sa structure}

Dans notre modèle, il nous faut distinguer la population par catégorie professionnelle afin de tenir compte de la variété des régimes de retraite en France : cadres, non cadres et fonctionnaires $(c \in\{c a, n c, f\})$. La taille de la population du type $(g, s, c)$ à la date $t$ est donc donnée par $N(t, g, s, c)$. Chaque catégorie d'individu est représentée, à chaque date, par un individu représentatif. Ce dernier est ainsi à un même instant employé-chômeur-inactif.

L'âge de fin d'études $D_{2}(g, s, c)$ joue un rôle important dans le modèle. En effet, l'âge de fin d'études, combiné à l'âge de liquidation de la retraite, détermine la durée moyenne d'activité qui intervient dans le calcul des pensions. La détermination de l'âge moyen de fin d'études est basée sur les statistiques d'Eurostat. La durée des études s'allonge pour les générations nées avant 1975 puis se stabilise pour les générations suivantes (Figure 2). Nous avons ensuite distingué l'âge de début d'activité selon le statut professionnel.

Figure 2 - Age de fin d'étude, durée de la carrière et de la retraite

(a) Hommes

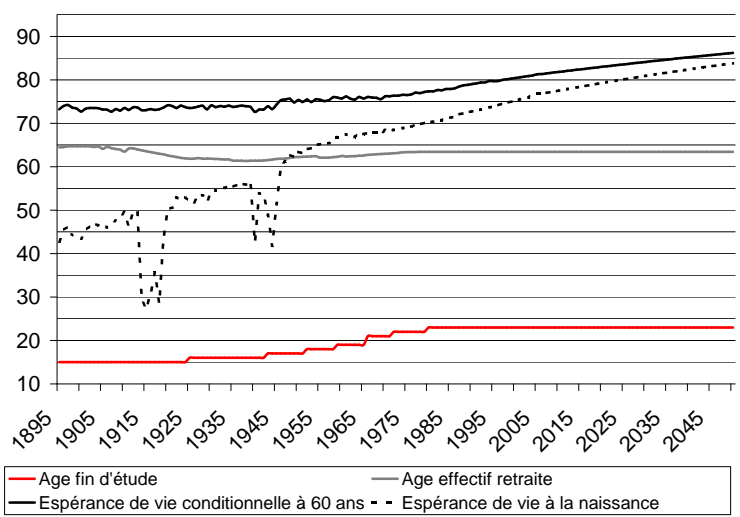

(b) Femmes

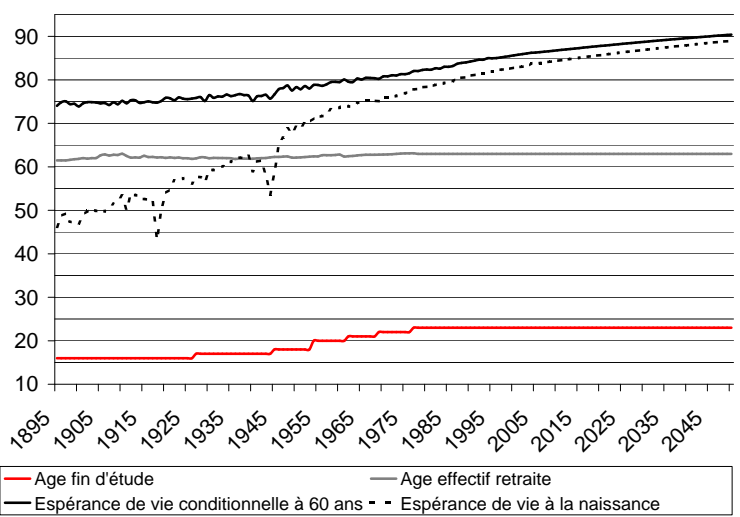

source : Calculs des auteurs

La participation des individus au marché du travail est considérée comme exogène dans le modèle et repose sur des données de l'Insee (Figure 3). Les hypothèses prospectives sur les niveaux d'activité sont optimistes : elles impliquent une augmentation de l'âge moyen de cessation d'activité de 3 ans à l'horizon 2040 ainsi qu'à une hausse significative des taux d'activités des seniors.

En affectant les parts de population et les taux d'activité par catégorie socio-professionnelle, l'objectif est de reproduire les principales caractéristiques de la population active passée et actuelle, $N_{a c t}(t, g, s, c)$. Nous avons ainsi cherché à reproduire les faits suivants : une proportion de $22 \%$ de cadres dans l'emploi privé total, en $2005^{3}$; une hausse progressive du taux d'encadrement au sein de l'emploi total du secteur privé, rapide entre 1950 et 1993 puis plus lente

3. Ce chiffre est basé sur la proportion de cotisants à l'AGIRC auxquels on rajoute les cotisants aux tranches supérieurs d'autres régimes en points (Ircantec, etc.) tels qu'ils ressortent de l'enquête EIR (échantillon interrégimes 
FigURE 3 - Taux d'activité par âge en 2000, 2015 et 2050

(a) Hommes

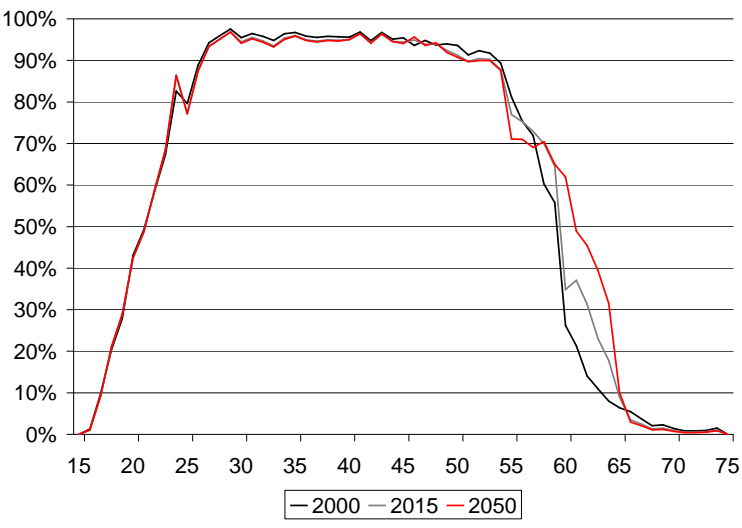

(b) Femmes

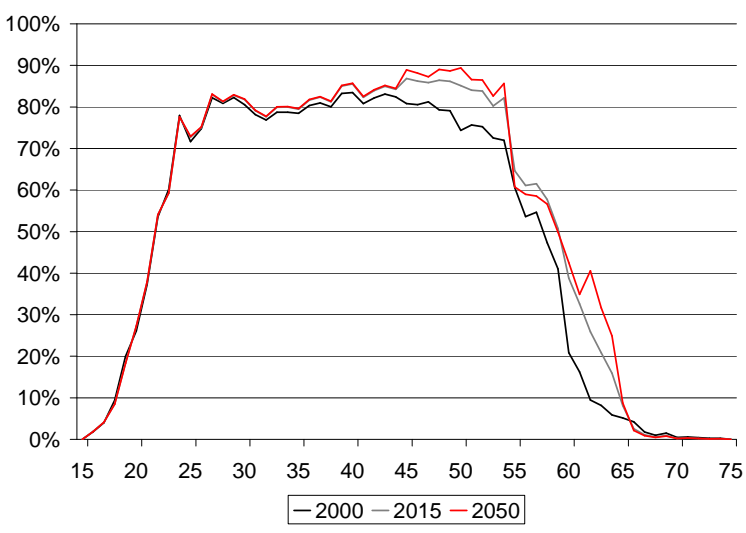

source : Insee

depuis 1993, soit une hausse de $12,1 \%$ en 1971 à $21 \%$ en 2000 puis $25 \%$ en 2040 ; une proportion croissante de femmes dans la population des cadres atteignant 32,7\% en 2005 (Chiffre Agirc mais aussi Ircantec); des recrutements massifs de fonctionnaires entre 1975 et 1985 puis une stabilisation voire une légère régression par la suite des embauches de fonctionnaires (Marchand, Massal \& Raynaud (2002)) ; en 2005, les fonctionnaires titulaires représentent $15,6 \%$ de l'emploi total, et 56,6\% d'entre eux sont des femmes ${ }^{4}$.

\subsection{Les retraités}

Dans le modèle on définit les retraités, $N_{\text {ret }}(t, g, s, c)$, comme l'ensemble des individus touchant une pension de retraite. Autrement dit, tous les individus d'un âge supérieur à 75 ans et tous les individus de chaque classe qui sont inactifs au-delà de 60 ans. Cependant, dans la réalité, de nombreuses pensions de retraite, mais aussi de nombreuses autres allocations visant à rétribuer la cessation anticipée d'activité sont effectivement versées. Nous avons donc supposé que certains individus passaient entre l'activité et la retraite par une phase de pré-retraite. Nous avons ainsi supposé qu'à partir de 56 ans et jusqu'à l'âge légal de départ à la retraite, toute réduction de l'activité d'une génération entre deux dates successives correspond à de la pré-retraite :

$$
N_{\text {pre }}(t, g, s, c)=\sum_{56<a(t, g)<60}\left(N_{a c t}(t-1, g, s, c)-N_{a c t}(t, g, s, c)\right) \text { pour c } \neq \text { "f" }
$$

Cette loi d'évolution quoique totalement ad hoc a le mérite de reproduire un nombre correct de pré-retraités en 2008 et une décroissance naturelle de leur nombre du fait de l'accroissement des taux d'activité des seniors.

des retraités)

4. Ministère de la Fonction Publique. 
Dans la précédente version du modèle (Chateau et al. (2009) et Chojnicki \& Magnani (2008)), nous faisions l'hypothèse que tous les individus d'une génération partaient à la retraite à une date donnée représentant l'âge moyen de départ à la retraite. A présent, nous considérons qu'à l'intérieur d'une génération les individus peuvent se retirer à des âges différents. Cet aspect est tout particulièrement important afin de calculer correctement la valeur des pensions servies lorsque les règles déterminant cette valeur à la liquidation sont non linéaires (du fait notamment des mécanismes de décote et surcote).

Afin de déterminer le nombre d'individu d'une génération partant à la retraite à un âge donné, nous supposons qu'à partir de 60 ans, toute réduction du taux d'activité correspond à un départ à la retraite. Ainsi, en utilisant les taux d'activité de l'Insee (et leur projection sur l'horizon d'étude), nous calculons pour chaque génération la fraction d'individus liquidant leur retraite entre 60 et 74 ans. Il convient de signaler que l'âge moyen de départ à la retraite pour une génération est de ce fait toujours considéré comme exogène (Figure 2). Pour déterminer la pension des individus qui liquident ou vont liquider leur pension dans les années à venir, nous avons reconstruit les carrières sur base de la structure par âge et par sexe des taux d'activité de 1950 à 2000.

La répartition par catégorie socio-professionnelle du stock actuel de retraités a été évaluée en s'appuyant sur différentes sources. A partir des données de l'échantillon inter régimes des retraités (IER), nous avons déterminé la répartition des retraités en fonctionnaires, cadres et non cadres. Nous avons évalué la répartition des cadres retraités entre hommes et femmes sur la base des données de l'AGIRC. Une partie des cotisants à l'IRCANTEC est rattachée au groupe "cadre". Sur la base de ces données nous avons alors ajusté les proportions de retraités calculées par notre modèle en 2005 qui découlaient des hypothèses précédentes en supposant une mortalité différente des individus selon la catégorie professionnelle. Nous avons de plus supposé que ces écarts d'espérance de vie perdureraient dans l'avenir. L'ensemble de ces hypothèses nous permettent d'obtenir par simulation les chiffres suivants pour 2005 :

- 12,8 millions de retraités sur un total de 13,2 millions d'individus âgés de plus de 60 ans. A noter que notre chiffre diffère de l'évaluation du COR (13,8 millions de retraités) notamment du fait de la non prise en compte des possibilités de cumul emploi-retraite et de la présence de poly pensionnés,

- 14,6\% des retraités sont des fonctionnaires (Ministère de la Fonction Publique).

- $12 \%$ de l'ensemble des retraités sont des anciens cadres et $20 \%$ des cadres retraités du secteur privé sont des femmes (Agirc).

\section{LA STRUCTURE DU MODÈLE}

Un reproche couramment adressé aux exercices usuels de projection des retraites est de ne s'en tenir qu'à une vision comptable et en équilibre partiel, en ne prenant en compte ni l'incidence des changements démographiques sur l'équilibre économique ni les effets des choix retenus en matière de retraite sur ce même environnement économique, tels que l'incidence sur les mar- 
chés des capitaux. Pour cette raison, nous développons ici une approche caractérisée par des interactions entre l'évolution démographique, la gestion du système de retraite, l'équilibre du marché du travail et l'équilibre macroéconomique. Une telle approche bouclée paraît indispensable pour traiter de la question de la réforme du système de retraite français et distingue ce travail de la plupart des autres études.

\subsection{Le secteur productif}

Dans le modèle OLGAMAP, le secteur productif est composé d'une entreprise représentative supposée agir de façon parfaitement concurrentielle. Elle produit un bien unique qui servira de support, soit à la consommation, soit à l'accumulation de capital physique. La production $Y_{t}$ au cours de la période $t$ s'effectue au moyen d'une combinaison de capital physique installé $K_{t-1}$ à la date $t$ et des services du facteur travail $N_{t}$ à l'aide d'une fonction de production de type Cobb-Douglas à rendements d'échelle constants :

$$
Y_{t}=K_{t-1}^{\alpha}\left(\Gamma_{t} N_{t}\right)^{1-\alpha}
$$

où $\alpha$ est la part de la rémunération du capital dans la valeur ajoutée. Le niveau de connaissance dans l'économie $\Gamma_{t}$ est supposé croître à taux exogène. A partir de 2010, ce paramètre est fixé à un taux de 1,8\% par an ce qui est compatible avec le scénario central des projections du Conseil d'Orientation des Retraites (2010a). Avant 2010, ce paramètre est calibré de façon à reproduire les taux de croissance effectifs de l'économie française.

La maximisation du profit en univers concurrentiel requiert l'égalisation des coûts réels des facteurs à leurs productivités marginales :

$$
\begin{aligned}
\frac{\partial Y(t)}{\partial K(t-1)} & =r_{K}(t)+\delta \\
\frac{\partial Y(t)}{\partial N(t)} & =w(t)
\end{aligned}
$$

où $\delta$ désigne le taux de dépréciation du capital physique, $r_{K}(t)$ le taux de rendement du capital physique et $w(t)$ le taux de salaire réel.

Nous devons préciser ici que les cotisations sociales payées par les employeurs ne sont pas explicitement introduites : nous faisons donc l'hypothèse que toutes les cotisations sociales sont payées par les salariés. Le paramètre $\alpha$ de la fonction de production est supposé constant et égal à $0,4 \%$. Nous supposons que $\delta=5,5 \%$ par an de sorte à reproduire un taux d'intérêt des obligations d'Etat $r_{\text {bond }}=3,5 \%$ en $2000^{5}$, chiffre qui correspond aux valeurs de long terme de d'Autume \& Quinet (2001).

5. Voir section 5.2 pour la distinction entre le rendement du capital physique $r_{K}(t)$ et celui des titres de la dette publique $r_{\text {bond }}(t)$ 


\subsection{Salaire et chômage d'équilibre}

La quantité de travail employé, $N_{t}$, dépend, d'un côté, de l'évolution de la population active découlant du bloc socio-démographique et, de l'autre, du niveau de salaire. Suivant d'Autume \& Quinet (2001), on adopte une approche WS-PS pour déterminer les niveaux de salaires réels et de chômage d'équilibre au niveau agrégé. On suppose qu'il existe des négociations salariales entre l'entreprise et un syndicat (représentant les intérêts des salariés) qui conduisent à appliquer une marge sur un salaire de réserve qui croît au même rythme que la productivité globale des facteurs, $\Gamma_{t}$, afin de garantir à long terme l'existence d'un taux de chômage structurel. Cette marge est, en outre, supposée décroître avec le niveau du taux de chômage moyen dans l'économie, traduisant de ce fait une érosion du pouvoir de négociation effectif des syndicats en cas de pénurie de demande de travail. Le salaire réel est alors déterminé de la manière suivante :

$$
\log (w(t))=0.5 \cdot \log (w(t-1))+0.5\left[a_{u} \bar{\theta}_{c h o}(t)+\log (\Gamma(t))+\Lambda(t)\right]
$$

où $\theta_{\text {cho }}(t)$ est le taux de chômage moyen dans l'économie. $\Lambda(t)$ est un paramètre d'ajustement étalonné, durant la phase de transition, de façon à reproduire notamment les taux de chômage historiques sur la période 2000-2009 ainsi qu'à assurer une convergence d'ici à 2020 du taux de chômage effectif vers un taux de chômage cible de $7 \%$ conformément au scénario pessimiste du Conseil d'Orientation des Retraites (2010a). $a_{u}$ est l'élasticité de long terme du salaire réel super brut au taux de chômage. Dans les simulations, on posera $a_{u}=-1$ ce qui revient à supposer une élasticité de long-terme unitaire du coût du travail au taux de chômage.

\subsection{Les comportements individuels}

\subsubsection{Profils de chômage, de salaire et revenus}

Comme nous l'avons mentionné précédemment, la population active découlant du bloc sociodémographique est exogène et dépend, à une date donnée, du nombre d'individus actifs par âge, genre et catégorie professionnelle. L'imperfection du marché du travail détermine un taux de chômage au niveau national qui est ensuite réparti par âge, genre et catégorie professionnelle par application de profils individuels constants.

A chaque date, l'individu représentatif de type $(g, s, c)$ reçoit un salaire moyen $w_{\text {rep }}(t, g, s, c)=$ $w(t, g, s, c)\left(1-\theta_{\text {cho }}(t, g, s, c)\right) \theta_{\text {act }}(t, g, s, c)$ où $\theta_{\text {cho }}$ et $\theta_{\text {act }}$ désignent respectivement le taux de chômage et le taux d'activité de ce type d'individu et $w_{\text {rep }}$ est le taux de salaire brut. Le taux de chômage individuel dépend alors de l'âge, du genre et de la catégorie professionnelle considérée. Le profil de salaire est simplement donné par le produit d'une prime d'expérience par âge et d'une prime de qualification par genre. Le calibrage de ces primes et donc du profil de salaire par âge, genre et profession est basé sur les données de l'enquête emploi de l'Insee.

A chaque date, le revenu d'un individu est la somme de ses revenus financiers et de ses revenus du travail. Avant l'âge légal de la retraite (60 ans dans le scénario de base), l'individu touche un 
revenu salarial, différencié selon l'âge, le genre et la catégorie professionnelle d'appartenance, et parfois une préretraite. Après cet âge, et jusqu'à 75 ans, l'individu perçoit pour partie des revenus salariaux et pour partie une pension de retraite calculée selon la législation. Au-delà, il ne touche plus que sa pension. Le revenu salarial est assujetti aux taux de cotisation retraite variants selon la catégorie professionnelle.

\subsubsection{Comportement de consommation et d'épargne}

La consommation courante de l'individu, comprenant aussi le coût des enfants, dépend, d'un côté, de son revenu disponible net courant, $\operatorname{Inc}(t, g, s, c)$ et, de l'autre, suivant d'Autume \& Quinet (2001), de sa richesse, $A(t-1, g, s, c)$, accumulée au début de la période :

$c(t, g, s, c)=(1-s(t, g))(1-\widetilde{\tau}(t))[r(t) A(t-1, g, s, c)+\operatorname{Inc}(t, g, s, c)]+c_{A} A(t-1, g, s, c)$

avec $s(t, g)$ la propension exogène à épargner le revenu disponible net, indépendante de la catégorie professionnelle, $c_{A}$ la propension exogène à consommer la richesse accumulée et $\widetilde{\tau}(t)$ un taux proportionnel uniforme d'impôt sur les revenus (du travail et du capital), visant à financer les pré-retraites et une partie des pensions des fonctionnaires. Une fraction constante, mais variant avec l'âge, du revenu disponible courant est épargnée. L'accumulation de patrimoine de l'individu se déduit alors de façon résiduelle comme la proportion du revenu disponible non consommé, ou non consacré à l'éducation des enfants, à laquelle s'ajoute éventuellement un héritage. Aussi les comportements d'épargne et de consommation diffèrent suivant les phases de cycle de vie. Les propensions marginales à épargner et à consommer la richesse sont calibrées de façon à reproduire un profil d'accumulation de patrimoine au cours du cycle de vie (Brender \& Gabrielli (2001)) et un montant agrégé d'héritage conformes à l'observation (Arrondel \& Laferrère (2001)). Cette modélisation de la consommation courante nous permet de reproduire un profil d'accumulation de la richesse décroissant à partir d'un certain âge (Figure 4) et ce sans supposer des propensions marginales à épargner négatives aux âges élevés qui apparaissent clairement contrefactuelles en France.

La contrainte budgétaire instantanée des ménages prend la forme usuelle suivante :

$$
\begin{array}{r}
A(t, g, s, c)+c(t, g, s, c)(1+\operatorname{cout}(t, g, s, c))= \\
{[1+(1-\widetilde{\tau}(t)) \cdot r(t)] A(t-1, g, s, c)+(1-\widetilde{\tau}(t)) \operatorname{Inc}(t, g, s, c)+h(t, g, s, c)}
\end{array}
$$

où $h(t, g, s, c)$ est l'héritage reçu par l'agent ${ }^{6}$. La variable cout $(t, g, s, c)$ exprime l'équivalent en termes de consommation d'un individu adulte et autonome de la consommation des enfants à charge des adultes, calibré à l'aide de l'étude de Hourriez \& Olier (1997).

6. Afin de faciliter la résolution numérique du modèle, on suppose ici simplement à l'instar de Imrohoroglu (1998), que les legs sont taxés à 100\% par l'Etat et redistribués de façon forfaitaire et uniforme à tous les individus survivants d'âge adulte. 
FIGURE 4 - Niveau de richesse par âge, genre et catégorie professionnelle (en euros)

(a) Homme

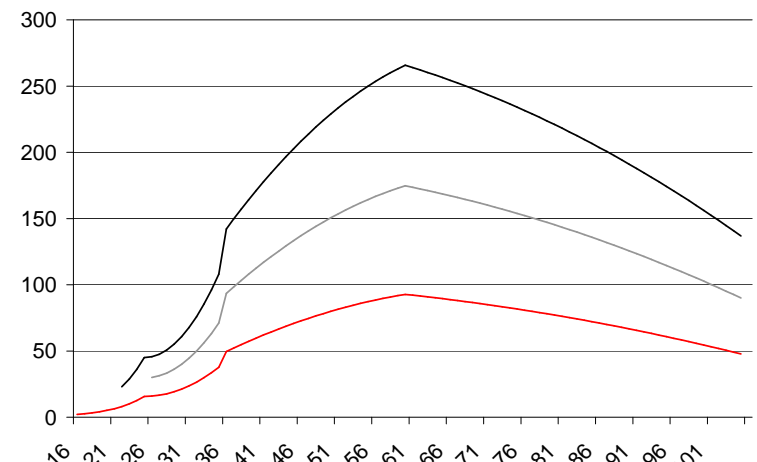

-Cadre-NonCadre - Fonction (b) Femme

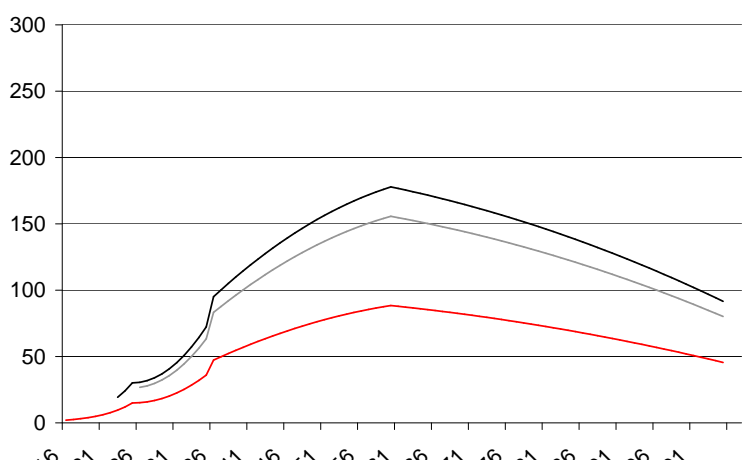

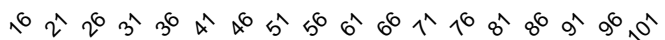

- Cadre - NonCadre - Fonction

source : Calculs des auteurs

\section{LES PENSIONS DE RETRAITE PUBLIQUES}

Le modèle porte sur les régimes de retraite les plus importants et couvre la quasi totalité des dépenses de retraite. Il s'agit des dépenses de retraites du secteur privé (pension de base et complémentaire), du secteur public et des non-salariés. Ces dépenses sont regroupées en plusieurs caisses : le régime général qui représente les dépenses de retraites de base autant pour les retraités du secteur privé que pour les non-salariés, deux caisses complémentaires selon que les individus du secteur privé qui y sont affiliés sont cadres ou pas, et une caisse de la fonction publique (y compris Cnracl). A la différence des deux autres régimes dont les ressources proviennent seulement des cotisants, une partie du produit de la taxe portant sur l'ensemble des revenus de la population adulte alimente aussi la caisse "fonction publique". Le fonctionnement de chacun des régimes tient compte des réformes de 1993, 2003 et 2008 mais n'intègre pas les effets de la réforme de 2010, celle-ci n'étant pas encore totalement définie au moment de la rédaction de cette étude.

\subsection{Le régime des fonctionnaires ("rf")}

A chaque période, la pension à la liquidation $P_{L}(t, g, s, " f$ ") versée par la caisse publique à un nouveau retraité fonctionnaire d'âge $a(t, g)$, compris entre 60 et 74 ans, est donnée par la formule suivante :

$$
\begin{aligned}
& P_{L}(t, g, s, " \mathrm{f} ")_{60 \leq a(t, g) \leq 74}= \\
& \pi_{r f}(t, g) \cdot\left(1-\operatorname{dec}_{r f}(t, g) \cdot \operatorname{Max}\left(0, \operatorname{Min}\left(65-a(t, g), D_{1}\left(g, s, " \mathrm{f}^{\prime \prime}\right)-a(t, g)+D_{2}(g, s, " \mathrm{f} ")\right)\right)\right) \cdot \\
& \left(1+\operatorname{sur}_{r f}(t, g) \cdot \operatorname{Max}\left(0, \operatorname{Min}\left(a(t, g)-60, a(t, g)-D_{2}(g, s, \text { "f" })-D_{1}(g, s, \text { "f" })\right)\right)\right. \text {. } \\
& w\left(t-1, g, s, \text { "f") } \operatorname{Min}\left(D_{1}(g, s, " \mathrm{f} "), a(t, g)-D_{2}(g, s, \text { "f" })\right)\right.
\end{aligned}
$$


où $\pi(t, g, " f$ " $)$ est le ratio de remplacement du dernier revenu du travail de l'individu représentatif $(w(t-1, g, s, " f "))$ égal à $75 \%$ dans le cas d'une carrière complète. $D_{1}(g, s, " f$ ") est la durée minimum de cotisation au régime des fonctionnaires pour obtenir une pension à taux plein (164 trimestre pour la génération 1952). $\operatorname{dec}_{r f}(t, g)$ est la pénalité pour cessation d'activité anticipée $^{7} . s_{r f}(t, g)$ est le coefficient de surcote appliqué pour toute année supplémentaire de cotisation au-delà de 60 et avant 65 ans lorsque les conditions d'assurance sont remplies (le taux est de 3\% par an).

\subsection{Le régime de base ("rb")}

Nous supposons que tous les non fonctionnaires sont affiliés au régime général qui est un régime par annuités à prestations définies. Le montant de la première pension que reçoit le retraité est calculé à partir du salaire de référence, $W_{R}(t, g, s, c)$, qui résume la carrière passée du futur prestataire. Le salaire de référence d'un individu qui liquide sa retraite à la date $t$ est la moyenne des revenus salariaux $w(t, g, s, c)$ sur les $A n(g)$ dernières années de la carrière ${ }^{8}$ :

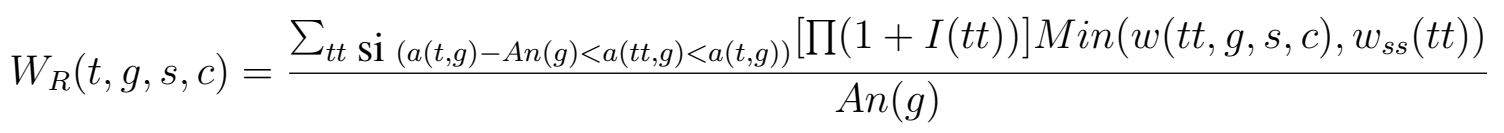

où $I(t)$ est l'indice de revalorisation du salaire reçu à la date $t$ au moment de la liquidation de la retraite, égal au taux d'inflation. $w_{s s}(t)$ est le plafond de la sécurité sociale, soit le seuil au-delà duquel aucun droit n'est acquis, conformément à la pratique en vigueur ce plafond de la sécurité social est ici proportionnel au revenu salarial moyen des non fonctionnaires :

$$
w_{s s}(t)=a_{p l a f}(t) \cdot \frac{\left.\sum_{(g, s, c) ; c \neq " ~ f "} N_{a c t}(t, g, s, c) \cdot w(t, g, s, c)\right) \cdot\left(1-\theta_{\mathrm{cho}}(t, g, s, c)\right)}{\sum_{(g, s, c) ; c \neq " ~} f^{\prime \prime} N_{a c t}(t, g, s, c) \cdot\left(1-\theta_{\mathrm{cho}}(t, g, s, c)\right)}
$$

Nous cherchons à reproduire au mieux les pensions effectives moyennes calculées sur la base des données de l'enquête inter-régime. Le coefficient de proportionnalité $a_{p l a f}(t)$ a été fixé à 1,05 suivant le rapport au salaire brut moyen des salariés des entreprises. Conformément à la législation en vigueur, le montant de la pension $P_{L}(t, g, s, c)$ au moment de la liquidation est donné, à la date $t$, par (si $c \neq " f ")$ :

7. La réforme d'Août 2003 a introduit une telle décote pour la fonction publique à partir du 1er janvier 2006. Son taux a progressivement augmenté pour finalement atteindre $5 \%$ en 2009 . La décote consiste à minorer la pension de retraite liquidée de 1,25\% par trimestre manquant pour atteindre, soit la durée d'assurance nécessaire pour bénéficier du taux maximum de liquidation, soit la limite d'âge, dans la limite de 20 trimestres.

8. Dans la pratique, il s'agit des 25 meilleures années à partir de la génération née en 1948. 


$$
\begin{array}{r}
{\frac{P}{\mathrm{~L}_{R}}(t, g, s, c)}_{W_{R}(t, g, s, c)}=\pi_{r b}(t, g) \\
\left(1-\operatorname{dec}_{r b}(t, g) \cdot \operatorname{Max}\left(0, \operatorname{Min}\left(65-a(t, g), D_{1}(g, s, c)-a(t, g)+D_{2}(g, s, c)\right)\right)\right) \cdot \\
\left(1+s u r_{r b}(t, g) \cdot \operatorname{Max}\left(0, \operatorname{Min}\left(a(t, g)-60, a(t, g)-D_{2}(g, s, c)-D_{1}(g, s, c)\right)\right)\right) \cdot \\
\operatorname{Min}\left(1, \frac{a(t, g)-D_{2}(g, s, c)}{\operatorname{pro}(t, g)}\right)
\end{array}
$$

où $\pi_{r b}(t, g)$ est le ratio de remplacement du salaire de référence (égal à $50 \%$ si les conditions d'âge et de durée d'assurance sont remplies par l'individu). $d e c_{r b}(t, g)$ est la pénalité pour toute cessation d'activité anticipée. Elle est fonction de la génération concernée, conformément à la législation en vigueur pour la CNAV et aux réformes récentes (cette décote varie de 2,5\% pour les générations nées avant 1944 à $1.25 \%$ pour les générations nées après 1952). Le taux de surcote $\operatorname{sur}(t, g)$ est fonction du nombre de trimestres cotisés après 60 ans et au-delà de la durée d'assurance nécessaire pour obtenir le taux plein. Ce taux dépend également de la date d'effet de la pension. pro $(t, g)$ est la durée exigée pour obtenir le taux plein. Elle sera portée, suivant la réforme d'août 2003, progressivement de 150 à 164 trimestres en 2012.

\subsection{Les régimes complémentaires ("rc")}

Les régimes complémentaires sont en pratique des régimes par points à cotisations définies. En France, les cadres cotisent à deux caisses distinctives selon que leur salaire dépasse ou non le plafond de la sécurité sociale. Les non cadres quant à eux cotisent à une unique caisse mais les règles sont différentes selon la partie du salaire au-dessus et en-dessous du plafond. Les caisses complémentaires fonctionnent avec un régime par points. Les individus de type $(g, s, c)$ achètent chaque année $t$ de leur vie active point $(t, g, s, c$, caisse) points auprès de la caisse à laquelle ils sont affiliés à un prix $P_{p} t(t, c)$ et les vendent à un prix $V(t, c)$ au moment où ils liquident leurs retraites. Plutôt que de distinguer deux caisses selon la CSP nous avons choisi de distinguer deux types de points accumulés :

$$
\begin{aligned}
\text { point }_{1}(t, g, s, c) & =\tau_{1}(c) \cdot \frac{\operatorname{Min}\left(w(t, g, s, c), w_{s s}(t)\right.}{P_{p t}(t, c)} \\
\text { point }_{2}(t, g, s, c) & =\tau_{2}(c) \cdot \frac{\operatorname{Max}\left(0, w(t, g, s, c)-w_{s s}(t)\right)}{P_{p t}(t, c)}
\end{aligned}
$$

Notons qu'implicitement, et conformément à la législation en vigueur, des points sont acquis durant la période de cessation anticipée d'activité, même si aucune cotisation n'est présente en contrepartie. Ces avantages non contributifs en général sont financés par les caisses d'assurancechômage ou le budget général. Dans notre cas ils sont financés par la taxe sur les revenus. Le nombre de point accumulés depuis de début de la carrière est simplement égal à : 


$$
N P_{i}(t, g, s, c)=c c(s, c)_{i} \sum_{t t \operatorname{si}\left(D_{2}(g, s, c) \leq a(t t, g) \leq a(t, g)\right)} \operatorname{point}_{i}(t t, g, s, c) \quad \text { pour i }=1,2
$$

où les $c c(s, c)_{i}$ sont des coefficients correcteurs fixés, une fois pour toute, pour chaque $(s, c)$ de façon à rendre compte de l'acquisition de points reflétant des avantages non contributifs (chômage indemnisé, enfants, ...). Ces coefficients ont été étalonnés afin de reproduire les pensions de retraites complémentaires à la liquidation conformes aux données issues de l'EIR. La pension complémentaire totale $P_{r c}(t, g, s, c)$ est ensuite calculée à chaque date $t$, grâce à la formule suivante et ce quelque soit l'âge du pensionné :

$$
\begin{array}{r}
P_{r c}(t, g, s, c)_{60 \leq a(t, g) \leq 74}=\operatorname{Min}\left(1,1-\operatorname{dec}_{r c}(t, g) .\right. \\
\operatorname{Min}\left(\operatorname{Max}\left(D_{1}(g, s, s)-a(t, g)+D_{2}(g, s, c), 0\right), 65-a(t, g)\right) . \\
\left(V_{1}(t, c) \cdot N P_{1}(t, g, s, c)+V_{2}(t, c) \cdot N P_{2}(t, g, s, c)\right)
\end{array}
$$

avec $V_{i}(t, c)$ la valeur des points en euros constants 2000 calculée grâce aux données historiques fournis par les caisses complémentaires AGIRC, ARRCO et IRCANTEC jusqu'en 2008 et fixées par la suite (i.e. indexée sur le niveau général des prix) sauf mention contraire (i.e. scénario de baisse programmée des pensions,...). De façon plus générale nous avons utilisée les séries de salaire super brut à partir de 1950 des comptes de la Nation afin de de reproduire de façon correcte l'ensemble des droits de retraite acquis par les différents individus à la date initiale de la simulation.

Jusqu'ici, nous avons calculée la pension au moment de la liquidation dans le régime des fonctionnaires et le régime de base. La pension que la génération $g$ perçoit à la date $t$ dépend de la fraction d'individus appartenant à cette génération partie à la retraite en $t$, en $t-1$, etc. Par exemple, en 2005, la pension touchée par les retraités appartenant à la génération 1940 (ayant donc 65 ans) est déterminée en considérant qu'une fraction de ces retraités est partie à la retraite à 65 ans en 2005, une autre à 64 ans en 2004,..., une autre encore à 60 ans en 2000. Dans ce cas, la pension doit donc être déterminée comme une moyenne pondérée (en utilisant comme poids le paramètre fraction $(t, g, a g, s, c)$ ) des pensions calculées au moment de la liquidation, en appliquant la règle suivante :

$$
\left.\left.P(t, g, s, c, \text { caisse })_{60 \leq a(t, g) \leq 74}=\sum_{t t} \sum_{a g} \operatorname{fraction}(t, g, a g, s, c)\right) \cdot P_{L}(t-(a(t t, g)-60), g, s, c)\right)
$$

Après la liquidation, la pension est indexée sur la base de l'inflation ce qui implique qu'elle reste constante en termes réels. 


\section{DYNAMIQUE ET BOUCLAGE MACROÉCONOMIQUE}

Le capital physique utilisé dans la production domestique évolue dans le temps selon l'investissement net effectué dans la période, alors que la dette publique, représentée uniquement par la dette de la Sécurité Sociale, évolue sur la base des soldes de chaque caisse de retraite.

\subsection{Equilibre des caisses de retraite}

Les recettes des caisses privés sont supposées égales à la somme des cotisations de tous les salariés du privée en activité à cette date, tandis que les recettes de la caisse publique tiennent aussi compte de la taxe proportionnelle sur l'ensemble des revenus des agents à chaque date $t$ :

$$
\begin{aligned}
\operatorname{Rec}(" \mathrm{rb}) & =\sum_{(c \neq " \mathrm{f} ", g, s)} N_{a c t}(t, g, s, c) \cdot\left(1-\theta_{c h o}(t, g, s, c)\right) \cdot[\operatorname{cet}(c, " \mathrm{rb} ") \cdot w(t, g, s, c) \\
& \left.+\tau(" \mathrm{rb} ") \operatorname{Min}\left(w(t, g, s, c), w_{s s}(t)\right)\right] \\
\operatorname{Rec}(" \mathrm{rc} ") & =\sum_{(c \neq " \mathrm{f} ", g, s)} N_{a c t}(t, g, s, c) \cdot\left(1-\theta_{c h o}(t, g, s, c)\right) \cdot \operatorname{cet}(c, " \mathrm{rc} ") \cdot w(t, g, s, c) \\
& +\sum_{(c \neq " \mathrm{f} ", g, s)} N_{a c t}(t, g, s, c) \cdot\left(1-\theta_{c h o}(t, g, s, c)\right) \cdot \tau_{a p}(c) \cdot\left[\tau_{1}(c) \cdot \operatorname{Min}\left(w(t, g, s, c), w_{s s}(t)\right)\right. \\
& \left.+\tau_{2}(c) \cdot \operatorname{Max}\left(0, w(t, g, s, c)-w_{s s}(t)\right)\right] \\
\operatorname{Rec}(" \mathrm{rf} ") & =\sum_{(g, s)} N_{a c t}(t, g, s, " f ") \cdot w(t, g, s, \mathrm{f} ") \cdot \tau(" \mathrm{rf} ") \\
& +\widetilde{\tau}\left[\left(r(t) \cdot \sum_{(g, s, c)} N(t, g, s, c) \cdot A(t, g, s, c)\right)+(1-\alpha) Y(t)\right]
\end{aligned}
$$

Le revenu salarial moyen est assujetti à différents taux de cotisation retraite : $\tau$ (" $r b$ ") est le taux de cotisation au régime de base, $\tau_{a p}(c)$ est le taux d'appel identique pour les deux régimes complémentaires, $\tau_{1}(c)$ et $\tau_{2}(c)$ les taux de de cotisation aux régimes complémentaires, enfin cet $(c$, caisse $)$ sont des taux de contribution exceptionnelle qui sont fixés suivant la législation en vigueur et ne donnant pas de droits à des pensions.

A chaque date $t$, les dépenses des caisses de retraites sont simplement données par la somme des pensions versées :

$$
\begin{aligned}
\operatorname{Dep}(" \mathrm{rb} ") & =\sum_{(c \neq " \mathrm{f} ", g, s) ; a(t, g) \geq 60} N_{r e t}(t, g, s, c) P_{r b}(t, g, s, c) \\
D e p(" \mathrm{rc} ") & =\sum_{(c \neq " \mathrm{f} ", g, s) ; a(t, g) \geq 60} N_{r e t}(t, g, s, c) P_{r c}(t, g, s, c) \\
D e p(" \mathrm{rf} ") & =\sum_{(g, s) ; a(t, g) \geq 60} N_{r e t}(t, g, s, " \mathrm{f} ") P_{r f}(t, g, s, " \mathrm{f} ")
\end{aligned}
$$




$$
+\sum_{\left(c \neq " \mathrm{f}^{\prime \prime}, g, s\right) ; 56 \leq a(t, g)<60} N_{\text {pre }}(t, g, s, c) \pi_{\text {pre }} w(t, g, s, c)
$$

A chaque période, le déficit primaire d'une caisse de retraite est défini par la différence entre les dépenses et les recettes : Def(caisse, $t)=\operatorname{Dep}($ caisse, $t)-\operatorname{Rec}($ caisse, $t), \forall$ caisse $\in$ $\{r b, r f, r c\}$. Lors du calibrage, on cherche à reproduire le niveau historique des dépenses propres à chacune des caisses que l'on a construites (Tableau 2). Plus concrètement, le modèle reproduit pour l'année 2005 les valeurs suivantes : un ratio prestations vieillesse-survie sur PIB de $12,05 \%$ (Comptes de la Sécurité Sociale) ainsi que la répartition de ces dépenses entre les trois caisses suivante $: \operatorname{Dep}(" r f ")=44 \%, D e p(" r b ")=27 \%, D e p(" r c ")=29 \%{ }^{9}$. Pour coller à ces données, nous avons dû ajuster certaines variables : si les profils des stocks de pensions liquidées entre 2000 et 2005 par génération, sexe et CSP reproduisent pour chaque caisse ceux issus des données de l'EIR, à cette même date, les niveaux moyens des pensions liquidées par caisse et les taux de remplacement à la liquidation (ainsi que les valeurs des points) pour chaque type d'agent entre 2000 et 2005 sont quant à eux ajustés.

Nous reproduisons entre 2000 et 2005 les soldes (Def(caisse, $t)$ ) des différentes caisses de retraite. Pour rendre compatibles les sorties du modèle à cette hypothèse, nous avons choisi d'ajuster certains paramètres de la partie recettes des régimes sur la période en question : le taux de taxe $\tilde{\tau}(t)$ pour la caisse publique, le taux d'appel dans les régimes complémentaires $\left(\tau_{a p}(t, c)\right)$ et le taux de cotisation au régime de base $(\tau(t, " r b "))$.

Par la suite, il est possible d'envisager différents ajustements des paramètres pour assurer ou non l'équilibre primaire des caisses. Si l'équilibre primaire n'est pas assuré, les dépenses excédentaires devront être financées par endettement. Dans le cas contraire, elles seront capitalisées. Comme il n'y a pas lieu de supposer que les différentes caisses aient accès à des sources de financement externes différentes de celles des autres agents, la dette globale du système de retraite Dette $_{\text {ret }}(t)$ évolue de la façon suivante, avec $R(t)=1+(1-\tilde{\tau}(t)) r(t)$ :

$$
\operatorname{Dette}_{r e t}(t)=R(t) \text { Dette }_{r e t}(t-1)+\sum_{\text {caisse }} \operatorname{Def}(\text { caisse }, t)
$$

On suppose qu'initialement il n'y a pas de dette $\left(\right.$ Dette $\left._{r e t}(2000)=0\right)$. En revanche, on reproduit les déficits observés de chacune des caisses du système de retraite entre 2001 et 2005 par calibrage des différents paramètres de générosité des pensions versées.

9. Ces chiffres sont calculés sur la base des données des rapports sur les comptes de la Sécurité Sociale en tenant notamment compte de l'ensemble des dépenses des régimes en points des non-salariés du secteur privé dans la caisse complémentaire et l'ensemble des dépenses des régimes en annuités de ces mêmes individus dans le régime de base. Les prestations versées par les régimes spéciaux sont quant à elles regroupées dans le "régime public". 
TABLEAU 2 - Les caisses de retraites intégrées dans le modèle (2000-2005)

\begin{tabular}{rrrrrrr} 
& 2000 & 2001 & 2002 & 2003 & 2004 & 2005 \\
\hline Dépenses du régime de base ("rb") & $5,21 \%$ & $5,23 \%$ & $5,25 \%$ & $5,26 \%$ & $5,24 \%$ & $5,30 \%$ \\
Dépenses régimes complémentaires ("rc") & $3,05 \%$ & $3,06 \%$ & $3,07 \%$ & $3,11 \%$ & $3,17 \%$ & $3,26 \%$ \\
Dépenses régimes des fonctionnaires ("rf") & $3,43 \%$ & $3,54 \%$ & $3,51 \%$ & $3,50 \%$ & $3,51 \%$ & $3,50 \%$ \\
\hline Total des dépenses de retraite & $\mathbf{1 1 , 6 9 \%}$ & $\mathbf{1 1 , 8 4 \%}$ & $\mathbf{1 1 , 8 3 \%}$ & $\mathbf{1 1 , 8 7 \%}$ & $\mathbf{1 1 , 9 2 \%}$ & $\mathbf{1 2 , 0 5 \%}$ \\
\hline Recettes du régime de base ("rb") & $5,47 \%$ & $5,61 \%$ & $5,54 \%$ & $5,52 \%$ & $5,45 \%$ & $5,45 \%$ \\
Recettes régimes complémentaires ("rc") & $3,37 \%$ & $3,40 \%$ & $3,32 \%$ & $3,54 \%$ & $3,58 \%$ & $3,63 \%$ \\
Recettes régimes des fonctionnaires ("rf") & $3,43 \%$ & $3,43 \%$ & $3,41 \%$ & $3,39 \%$ & $3,39 \%$ & $3,37 \%$ \\
\hline Total des recettes & $\mathbf{1 2 , 2 8 \%}$ & $\mathbf{1 2 , 4 4 \%}$ & $\mathbf{1 2 , 2 7 \%}$ & $\mathbf{1 2 , 4 5 \%}$ & $\mathbf{1 2 , 4 1 \%}$ & $\mathbf{1 2 , 4 5 \%}$ \\
\hline Solde du régime de base ("rb") & $0,26 \%$ & $0,38 \%$ & $0,29 \%$ & $0,26 \%$ & $0,21 \%$ & $0,15 \%$ \\
Solde régimes complémentaires ("rc") & $0,32 \%$ & $0,34 \%$ & $0,25 \%$ & $0,43 \%$ & $0,41 \%$ & $0,37 \%$ \\
Solde régimes des fonctionnaires ("rf") & & $-0,12 \%$ & $-0,10 \%$ & $-0,11 \%$ & $-0,12 \%$ & $-0,13 \%$ \\
\hline Solde total & $\mathbf{0 , 5 8 \%}$ & $\mathbf{0 , 6 0 \%}$ & $\mathbf{0 , 4 4 \%}$ & $\mathbf{0 , 5 8 \%}$ & $\mathbf{0 , 4 9 \%}$ & $\mathbf{0 , 3 9 \%}$ \\
\hline \hline Sound
\end{tabular}

Source : Rapport à la commission des comptes de la Sécurité Sociale, Calculs des auteurs

\subsection{Equilibre du marché financier}

Le bouclage du modèle s'analyse à partir du fonctionnement du marché du capital. Les simulations sont ici réalisées en économie fermée, c'est-à-dire que le taux d'intérêt s'ajuste pour équilibrer le marché du capital au niveau national. Le bouclage du modèle est alors néo-classique : ex ante, l'offre nette de fonds prêtables des résidents, c'est-à-dire la richesse agrégée des ménages détermine le stock de capital installé dans le pays à la période suivante.

Nous supposons dans le modèle l'existence de deux types d'actifs financiers : le capital physique et les obligations d'Etat. Ces deux actifs, en économie fermée, sont financés par la seule richesse des ménages :

$$
K(t)+\operatorname{Dette}_{r e t}(t)=\Sigma_{(g, s, c)} N(t, g, s, c) A(t, g, s, c)
$$

Nous faisons l'hypothèse que les deux actifs sont parfaitement substituables. Le taux de rendement des obligations d'Etat coïncide avec le taux de rendement du capital physique, exception faite d'une prime de risque supposée constante :

$$
r_{\text {bond }}(t)=r_{K}(t)-\text { prime }
$$

Le rendement moyen du patrimoine $(r(t))$ est alors donné par une moyenne pondérée des rendements du capital physique et des obligations d'Etat, la pondération dépendant de la composition du portefeuille. Nous supposons que la composition des portefeuilles est identique pour chaque classe d'âge et qu'elle reflète la structure de l'offre totale d'actifs financiers.

L'équilibre du marché financier (équation 17) suffit à décrire l'équilibre général du modèle en économie fermée. En effet, la loi de Walras implique que l'équilibre du marché des biens et 
services est aussi assuré :

$$
Y=K(+1)-(1-\delta) K+\sum_{(g, s, c)}[(1+\operatorname{cout}(t, g, s, c)) \cdot c(t, g, s, c) \cdot N(t, g, s, c)]
$$

\section{EVALUATION À MOYEN TERME De L'ÉVOLUTION DU SYSTÈME DE RETRAITE}

Avant d'évaluer l'impact de l'introduction d'un nouveau système par comptes notionnels, nous présentons un scénario de référence décrivant les effets de moyen terme découlant du fonctionnement du système de retraite actuel. Celui-ci servira de point de comparaison aux différentes variantes simulées en comptes notionnels.

\subsection{Le scénario de référence}

Après 45 ans d'existence et plusieurs réformes visant à en accroître la générosité (création du minimum vieillesse en 1956, augmentation du taux de remplacement du régime général en 1971 ou abaissement de l'âge de départ à la retraite à 60 ans en 1982), le livre blanc sur les retraites de 1991 marque la prise de conscience du problème de financement à venir du système de retraite français. Depuis, plusieurs réformes se sont succédé. Parmi celles-ci, la réforme Balladur de 1993 a réduit la générosité globale du système général en augmentant progressivement la durée de cotisation pour bénéficier du taux plein (de 150 à 160 trimestres à raison d'un trimestre supplémentaire par an entre 1993 et 2003 ; paramètres $D 1(g, s, " c$ ") et $D 1(g, s, " n c ")$ du modèle), en augmentant le nombre d'années prises en compte dans le calcul du salaire de référence (de 10 à 25 ans entre 1993 et 2008 ; variable $W_{R}(t, g, s, c)$ du modèle) et en indexant les pensions et les salaires entrant dans le calcul du salaire de référence sur les prix, et non plus sur les salaires. Cette réforme a été étendue aux autres régimes (notamment ceux de la fonction publique) par la réforme Fillon de 2003 (qui prévoit notamment un allignement progressif de la durée de cotisation entre secteur privé et public, $D 1(g, s, " c ")=D 1(g, s, " n c ")=D 1(g, s, " f ") \forall t \geq 2008)$. Cette dernière prévoit, en outre, un mécanisme d'accroissement quasi-automatique de la durée légale de cotisation en fonction des gains d'espérance de vie, la durée de cotisation passant progressivement à 41,5 ans en 2020 .

Les résultats du scénario de référence (Tableau 3) montrent que, même complètement appliquée, la réforme Fillon de 2003 ne suffit pas à garantir la solvabilité des régimes de retraite. Dans le modèle, le besoin de financement (différence entre dépenses et recettes rapportées au PIB) croît progressivement avec les départs massifs des dernières générations du baby boom jusqu'à atteindre $3 \%$ en 2050, l'accumulation des déficits aboutissant à une dette des régimes de retraite supérieure à $70 \%$ du PIB à cet horizon. Selon nos projections, les dépenses de retraite devraient augmenter de 12,1\% du PIB en 2005 à $15 \%$ en 2030, puis à 15,9\% en 2050. Ces évolutions s'expliquent par la forte augmentation du nombre de retraités (de 13,2 millions en 2005 à 23,1 millions en 2050) alors que dans le même temps la masse des travailleurs est essentiellement constante (Tableau 1). Entre 2005 et 2050, on passerait ainsi d'environ 5 retraités pour 10 
travailleurs à plus de 8 retraités pour 10 travailleurs. Sur la même période, la part des dépenses de retraite dans le PIB augmenterait "seulement" de 3,8 points du fait de la baisse considérable $(-18 \%)$ du ratio de la pension moyenne des retraités au salaire moyen des actifs de la période, découlant de l'application des réformes récentes et en particulier de l'indexation des pensions sur les prix ${ }^{10}$.

Concernant les effets macroéconomiques, le déficit accru du système de retraite ralentit l'accumulation du capital. Jusqu'en 2020, la croissance économique reste soutenue dans le modèle ; puis elle ralentit, passant de $2 \%$ en 2020 à 1,5\% en 2050, du fait de l'évolution de l'investissement et de la stagnation de la population active. Malgré le ralentissement de l'accumulation du capital, le vieillissement démographique provoque une augmentation du capital par unité de travail efficace et donc une baisse du taux d'intérêt d'équilibre, ce qui permet d'amoindrir l'explosion de la dette du système de retraite.

Une description plus fine peut également être proposée selon les différentes caisses de retraite intégrées dans le modèle (Figure 5(a)). En particulier, l'accroissement des dépenses de retraite à l'horizon 2050 provient des régimes des salariés du privé (" $r b$ " et " $r c$ ") alors que la part des dépenses de la caisse du régime public tend à décroître après une légère augmentation jusqu'en 2020. De fait, l'évolution des besoins de financement pour chacune des caisses découle de celle des niveaux relatifs de dépenses. Ces évolutions par caisse s'expliquent en grande partie par les caractéristiques démographiques. Ainsi, le nombre de retraités augmente de presque $70 \%$ pour l'ensemble de la population alors qu'il n'augmente "que" de $58 \%$ dans le cas des salariés du secteur public et se retrouve multiplié par 3,5 s'agissant de la catégorie des cadres. Dès lors, même si le nombre de travailleurs dans la fonction publique tend à diminuer à l'horizon 2050 (de l'ordre de $15 \%$ alors qu'il augmente de près de $10 \%$ pour l'ensemble de la population), les caractéristiques de financement de la caisse des salariés de la fonction publique (en particulier le fait qu'elle soit en grande partie financée par une taxe portant sur l'ensemble de la population et pas seulement par une cotisation sur les seuls travailleurs du secteur public) expliquent cette absence de besoin de financement en 2050 .

Comme nous l'avons signalé, les réformes récentes tendent à réduire le niveau de générosité du système de retraite. Ainsi, les taux de remplacement (mesurés ici comme le rapport entre la première pension perçue et le dernier salaire) décroissent pour quasiment toutes les catégories d'ici à 2050 (Figure 5(b)). Plusieurs conclusions méritent d'être mises en évidence. D'une part, pour un âge de liquidation donné, les taux de remplacement sont clairement différenciés en fonction de la CSP, les cadres ayant les taux de remplacement les plus faibles (du fait du mécanisme du plafond de la sécurité sociale) et les fonctionnaires ceux les plus élevés (du fait de la prise en compte du seul dernier salaire dans le calcul de la pension). Les écarts entre CSP tendent à se réduire dans le temps, les réformes récentes aboutissant notamment à une convergence des

10. Malgré une méthodologie et un champ d'analyse différents, nos résultats apparaissent relativement comparables à ceux du scénario C du Conseil d'Orientation des Retraites (2010b) qui aboutit à l'horizon 2050 à un besoin de financement de l'ordre de $3 \%$ du PIB avec un taux de chômage cible à $7 \%$ mais un taux de croissance de la PGF de $1,5 \%$ par an (contre $1,8 \%$ ici). 
TABLEAU 3 - Comparaison du système de retraite actuel et du système par comptes notionnels

\begin{tabular}{|c|c|c|c|c|c|}
\hline & 2005 & 2020 & 2030 & 2040 & 2050 \\
\hline & \multicolumn{5}{|c|}{ Scénario de référence } \\
\hline Taux de croissance du PIB (en \%) & $1,9 \%$ & $2,0 \%$ & $1,7 \%$ & $1,7 \%$ & $1,5 \%$ \\
\hline Taux d'investissement brut (normalisé à 1 en 2005) & 1,00 & 0,97 & 0,93 & 0,89 & 0,82 \\
\hline Taux d'intérêt (normalisé à 1 en 2005) & 1,00 & 0,84 & 0,80 & 0,77 & 0,77 \\
\hline Dépenses de retraite (en \% du PIB) & $12,1 \%$ & $14,2 \%$ & $15,0 \%$ & $15,5 \%$ & $15,9 \%$ \\
\hline Besoin de financement (en \% du PIB) & $0,4 \%$ & $-1,4 \%$ & $-2,2 \%$ & $-2,6 \%$ & $-3,0 \%$ \\
\hline Dette des régim & $-2,8 \%$ & $6,2 \%$ & $22,9 \%$ & $45,4 \%$ & $72,7 \%$ \\
\hline \multirow[t]{2}{*}{ Pension moyenne/salaire moyen (normalisé à 1 en 2005) } & 1,00 & 0,94 & 0,87 & 0,83 & 0,82 \\
\hline & \multicolumn{5}{|c|}{ Scénario de référence - Economie ouverte } \\
\hline Taux & $1,9 \%$ & $2,0 \%$ & $1,8 \%$ & $1,9 \%$ & $1,9 \%$ \\
\hline d'investissement & 1,00 & 0,97 & 0,98 & 0,98 & 0,98 \\
\hline Taux d'in & 1,00 & 0,82 & 0,78 & 0,74 & 0,70 \\
\hline Dépe & $12,1 \%$ & $14,1 \%$ & $14,9 \%$ & $15,3 \%$ & $15,3 \%$ \\
\hline Besoin & $0,4 \%$ & $-1,3 \%$ & $-2,1 \%$ & $-2,4 \%$ & $-2,4 \%$ \\
\hline (B) & $-2,8 \%$ & $5,5 \%$ & $21,3 \%$ & $41,0 \%$ & $60,4 \%$ \\
\hline \multirow[t]{2}{*}{ Pension moyenne/salaire moyen (normalisé à 1 en 2005) } & 1,00 & 0,93 & 0,86 & 0,82 & 0,80 \\
\hline & \multicolumn{5}{|c|}{ Comptes notionnels (scénario A) } \\
\hline Tau & $1,9 \%$ & $2,0 \%$ & $1,7 \%$ & $1,7 \%$ & $1,6 \%$ \\
\hline Taux & 1,00 & 0,97 & 0,94 & 0,91 & 0,86 \\
\hline Taux d'il & 1,00 & 0,84 & 0,80 & 0,77 & 0,76 \\
\hline Dép & $12,1 \%$ & $14,1 \%$ & $14,9 \%$ & $15,2 \%$ & $15,2 \%$ \\
\hline IB) & $0,4 \%$ & $-1,4 \%$ & $-2,1 \%$ & $-2,4 \%$ & $-2,4 \%$ \\
\hline Dette des rég & $-2,8 \%$ & $6,3 \%$ & $22,3 \%$ & $43,1 \%$ & $65,5 \%$ \\
\hline \multirow[t]{2}{*}{ Pension moyenne/salaire moyen (normalisé à 1 en 2005) } & 1,00 & 0,95 & 0,87 & 0,83 & 0,80 \\
\hline & \multicolumn{5}{|c|}{ Comptes notionnels (scénario B) } \\
\hline Tal & $1,9 \%$ & $2,0 \%$ & $1,8 \%$ & $1,9 \%$ & $1,9 \%$ \\
\hline Taux d'investisseme & 1,00 & 0,98 & 0,97 & 0,98 & 0,98 \\
\hline Taux d & 1,00 & 0,84 & 0,80 & 0,77 & 0,76 \\
\hline o du PIB) & $12,1 \%$ & $14,0 \%$ & $14,2 \%$ & $13,9 \%$ & $13,4 \%$ \\
\hline Besoi & $0,4 \%$ & $-1,3 \%$ & $-1,4 \%$ & $-1,1 \%$ & $-0,6 \%$ \\
\hline e des rég & $-2,8 \%$ & $6,1 \%$ & $19,0 \%$ & $29,9 \%$ & $35,7 \%$ \\
\hline \multirow[t]{2}{*}{ Pension moyenne/salaire moyen (normalisé à 1 en 2005) } & 1,00 & 0,94 & 0,83 & 0,76 & 0,71 \\
\hline & \multicolumn{5}{|c|}{ Comptes notionnels (scénario C) } \\
\hline$\%)$ & $1,9 \%$ & $2,0 \%$ & $1,9 \%$ & $2,0 \%$ & $2,1 \%$ \\
\hline Taux d'investisseme & 1,00 & 0,98 & 0,99 & 1,04 & 1,08 \\
\hline alisé à 1 en 2005) & 1,00 & 0,84 & 0,80 & 0,78 & 0,76 \\
\hline Dépenses de retraite (en \% du PIB) & $12,1 \%$ & $14,0 \%$ & $13,6 \%$ & $12,7 \%$ & $11,7 \%$ \\
\hline Besoin de financement (en \% du PIB) & $0,4 \%$ & $-1,2 \%$ & $-0,8 \%$ & $0,1 \%$ & $1,1 \%$ \\
\hline Dette des régimes de retraite (en \% du PIB) & $-2,8 \%$ & $6,0 \%$ & $16,3 \%$ & $18,6 \%$ & $10,4 \%$ \\
\hline Pension moyenne/salaire moyen (normalisé à 1 en 2005) & 1,00 & 0,93 & 0,80 & 0,69 & 0,62 \\
\hline
\end{tabular}

Source : Calculs des auteurs 
durées de cotisation entre régimes. D'autre part, les taux de remplacement sont d'autant plus élevés que la liquidation de la pension intervient tard, ce phénomène étant encore accentué par l'introduction des mécanismes de décote et de surcote.

\section{FigURE 5 - Résultats du scénario de référence}

(a) Dépenses (Dep) et besoins de financement (Def) des régimes de retraites (en \% du PIB)

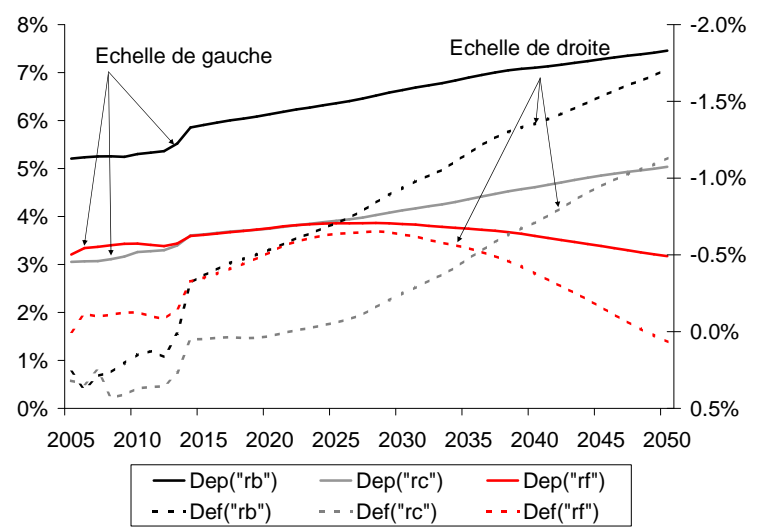

(b) Taux de remplacement (première pension/dernier salaire) selon l'âge de liquidation et la CSP

(2005-2050)

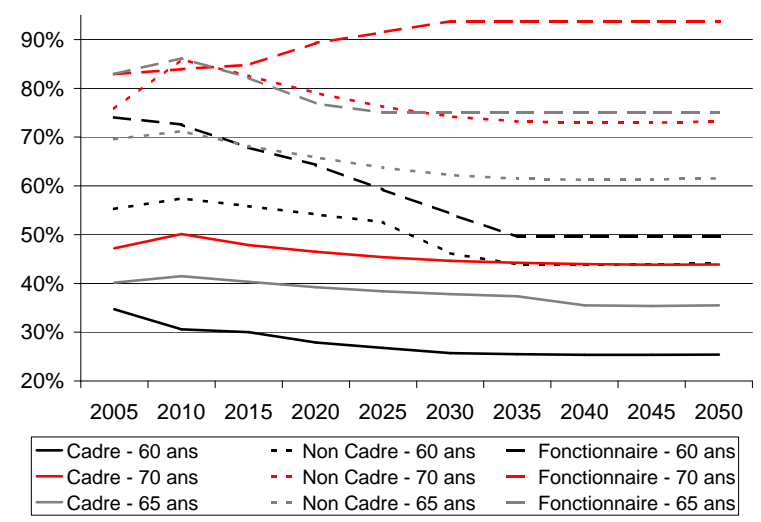

source : Calculs des auteurs

Pour assurer l'équilibre financier des régimes de retraite publics, une première voie consiste à réduire le montant des pensions versées, en maintenant les taux de cotisation à leur niveau prévu par les réformes récentes. Une seconde voie consiste, au contraire, à ajuster les taux de cotisation en conservant les règles actuelles de calcul des pensions ${ }^{11}$. Si les retraités devaient supporter tout le poids du rééquilibrage, cela signifierait en 2050 une baisse supplémentaire du ratio entre la pension moyenne et le salaire moyen de presque $20 \%$ soit une baisse de près de $35 \%$ par rapport à 2005 (Figure 6). Si l'équilibre portait entièrement sur les actifs, les taux de cotisation retraite devraient augmenter de l'ordre de 4,5 points $(+18 \%)$ d'ici à 2050. A titre de comparaison, l'abaque proposée par le Conseil d'Orientation des Retraites (2010a) suppose un équilibrage du système de retraite par une réduction de 36 à $38 \%$ du ratio pension moyenne/salaire moyen ou par un accroissement du taux de cotisation compris entre 3,5 et 7 points selon les hypothèses de croissance et de chômage considérées.

En définitive, la "solvabilité financière" du régime de retraite français telle qu'elle est souvent présentée dans les études et rapports publics dépend pour l'essentiel des hypothèses faites en matières de perspectives de croissance et de comportement d'activité. Cependant l'apport de notre démarche consiste à s'affranchir de ces purs calculs comptables afin d'analyser l'impact même des modes de financement des régimes de retraite, y compris par endettement, sur la dynamique de l'économie. L'effet retour est, de fait, d'autant plus important que l'économie

11. Ces réformes peuvent bien évidemment être panachées. 
considérée est tributaire des seuls capacités de financement de ses résidents ${ }^{12}$. Les simulations décrites jusqu'ici sont réalisées en économie fermée, ce qui implique que le taux d'intérêt s'ajuste pour équilibrer le marché du capital au niveau national. Cette hypothèse permet de prendre en compte une détente du taux d'intérêt lorsque le besoin de financement des régimes de retraite se réduit du fait des réformes. Elle néglige cependant la nature mondiale du vieillissement démographique, certes selon des calendriers différents dans les différentes régions du globe. En effet, le taux d'intérêt dans les décennies à venir sera influencé par la démographie mondiale, l'épargne étant fonction de la proportion de la population d'âge "mûr" selon l'hypothèse de cycle de vie. Par ailleurs, l'épargne nationale dégagée par une réforme des retraites ne se traduit pas par une baisse des taux d'intérêt en économie ouverte, mais par des sorties de capitaux qui réduisent le bénéfice attendu en termes d'accumulation de capital et de croissance. Afin d'évaluer l'importance du choix de l'environnement financier, le scénario de référence est également simulé en économie ouverte, en retenant comme taux d'intérêt mondial le profil intertemporel découlant des résultats du modèle INGENUE (2006).

INGENUE (2006) projette une baisse de la productivité marginale du capital au niveau mondial de 3 points de pourcentage entre 2005 et 2050 du fait du vieillissement de la population mondiale. Les résultats du scénario en économie ouverte sont plus optimistes que ceux du scénario de référence (tableau 3) car le taux d'intérêt français en économie fermée diminue moins que le taux d'intérêt mondial. Par conséquent, en économie ouverte, les capitaux affluent en provenance du reste du monde afin de bénéficier d'une rémunération supérieure, ce qui permet d'égaliser le taux d'intérêt français au taux mondial. En économie ouverte, la croissance du PIB est alors plus soutenue, ce qui se traduit par des dépenses et un déficit du système de retraite, relativement au PIB, inférieur d'environ 0,5 point par rapport au scénario de référence. Toutefois, ce scénario d'économie ouverte est lui-même discutable car il suppose une parfaite intégration financière au niveau mondial. Par ailleurs, la France est supposée être le seul pays à réaliser une réforme. Si l'on suppose maintenant que l'intégration financière se limite largement, de facto, aux économies avancées et que ces dernières seront toutes amenées à mettre en œuvre des réformes, c'est l'hypothèse d'économie fermée qui semble la plus raisonnable, ce que nous retenons dans les simulations qui suivent.

\subsection{Introduction d'un système par comptes notionnels}

Un système de comptes notionnels calcule la pension de façon à égaliser la valeur acquise des cotisations versées et la valeur actualisée des pensions reçues. Le principe de base de ce système est donc inspiré de la capitalisation, les cotisations acquittées chaque année étant virtuellement créditées sur un compte individuel, sauf que le système continue de fonctionner par répartition, ce qui lui en assure la stabilité en l'isolant de l'incertitude découlant du rendement des marchés

12. Il convient de signaler que les purs modèles comptables, tels que ceux utilisés par le Conseil d'Orientation des Retraites (2010a), impliquent l'hypothèse (très peu réaliste) que le taux d'intérêt mondial n'est pas affecté par le phénomène de vieillissement démographique qui, au contraire, concerne la plupart des pays développés (voir Börsch-Supan, Ludwig \& Winter (2006) et INGENUE (2006)). 
FiguRE 6 - Evolution du ratio pension moyenne/salaire moyen et du taux de cotisation avec et sans réformes supplémentaires $(=1$ en 2005)

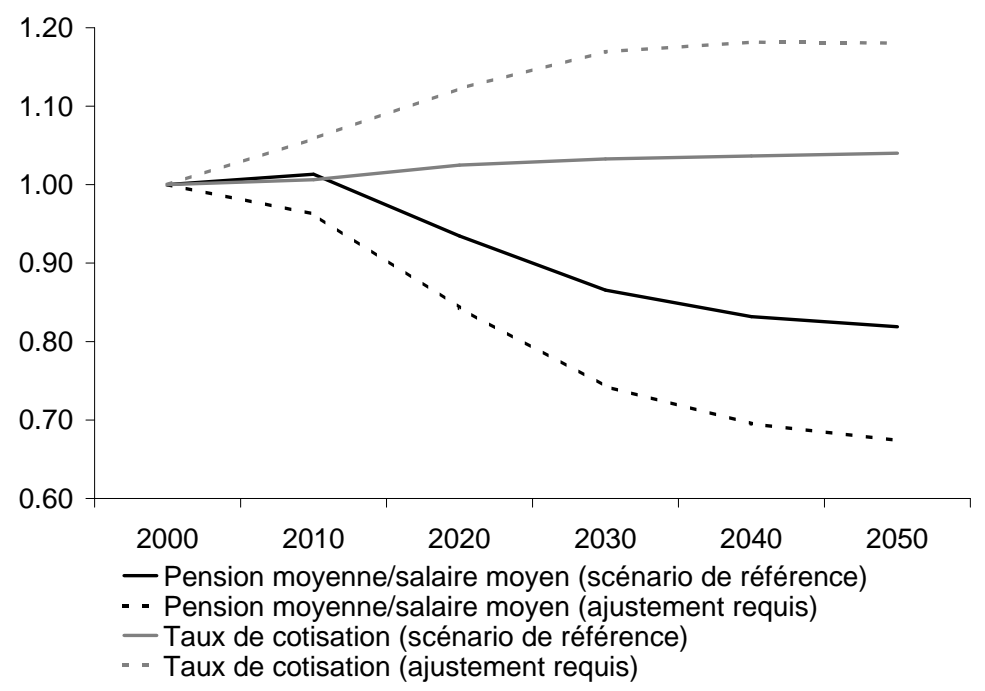

financiers. Nous simulons ici un premier scénario (A) avec mise en place progressive, entre 2015 et 2030, d'un système unique par comptes notionnels se substituant entièrement, à terme, à tous les régimes actuels (de base et complémentaires). Concrètement, cela revient à subtituer aux équations (8), (11) et (14), décrivant les règles de calcul actuelles des pensions respectivement dans le régime de la fonction publique, le régime de base et le régime complémentaire par la formule suivante :

$$
\begin{gathered}
P_{L}^{c n}(t, g, s, c)_{60 \leq a(t, g) \leq 74}= \\
\sum_{t t \text { si } D_{2} \leq a(t t, g) \leq a(t, g)} \tau_{c n} w_{r e p}(t t, g, s, c)\left(1+r_{w}(t t)^{a(t, g)-a(t t, g)}\right. \\
\sum_{t t \text { si } a(t t, g)>a(t, g)}\left(1+r_{\text {pens }}\right)^{a(t, g)-a(t t, g)} \cdot\left(1+r_{\text {index }}\right)^{a(t t, g)-a(t, g)} \cdot \operatorname{Pr}_{\text {survie }}(t t, g, s)
\end{gathered}
$$

où $a(t, g)$ désigne, comme précédemment, l'âge effectif de départ à la retraite (différentié entre 60 et 74 ans).

L'introduction d'un système de comptes notionnels nécessite plusieurs choix de la part du décideur politique :

- Le taux de cotisation $\tau_{c n}$ est ici supposé constant sur la période de projection. Il est fixé (à une valeur proche de $22 \%$ ) de manière à maintenir constante la masse des cotisations découlant de l'ancien système à la date de début de la transition :

$$
\sum_{\text {caisse }} \operatorname{Rec}(" 2015 ", \text { caisse })=\tau_{c n} \sum_{(g, s, c)} N_{a c t}(" 2015 ", g, s, c) \cdot\left(1-\theta_{\text {cho }}(" 2015 ", g, s, c)\right) \cdot w(" 2015 ", g, s, c)
$$


- Le taux de rendement appliqué aux cotisations passées, $r_{w}(t)$, constitue le deuxième paramètre essentiel du système. En considérant que le rendement implicite d'un système par répartition coïncide avec le taux de croissance de la masse salariale, et de sorte à garantir la stabilité financière du système, nous retenons ici, pour les cotisations versées à la date $t$, le taux de croissance de la masse salariale de la même période. Il est tout à fait envisageable de choisir d'autres références pour le taux de rendement à appliquer aux cotisations. Par exemple, en Suède, le taux retenu est le taux de croissance moyen des salaires; en Italie, il s'agit du taux de croissance moyen du PIB des cinq dernières années.

- Le taux d'actualisation des pensions retenu au moment de la liquidation, $r_{p e n s}$, constitue le troisième paramètre clé du nouveau système. Pour que le nouveau système soit viable, ce taux devrait correspondre au taux de croissance moyen des cotisations sur la période durant laquelle est versée la retraite. Ce dernier n'étant pas connu par anticipation, nous retenons une valeur de 1,8\% dans le scénario de base (scénario A). En Suède et en Italie, les taux retenus sont respectivement de $1,6 \%$ et $1,5 \%$.

- La règle d'indexation des pensions revient à opérer un choix entre (i) une indexation sur les prix ce qui revient à supposer une première pension relativement élevée puis un décrochage progressif du niveau des pensions par rapport à l'évolution des salaires et (ii) une indexation sur les salaires revenant à verser une première pension plus faible. Nous retenons ici l'hypothèse d'une indexation des pensions sur les prix comme dans le système actuel, ce qui implique que $r_{\text {index }}=0$. Ce choix n'a aucun impact au niveau de la soutenabilité financière du système mais n'est pas neutre d'un point de vue individuel du fait des fortes disparités d'espérance de vie.

- Les coefficients de conversion du capital virtuel en rente dépendent également de l'espérance de vie de la génération et de l'âge de départ à la retraite. Pour un capital virtuel donné, plus l'espérance de vie de la génération est élevée, plus la pension mensuelle sera faible ; et inversement plus l'âge effectif de départ à la retraite est élevé et plus la pension mensuelle le sera également. $P r_{\text {survie }}(t, g, s)$ est la probabilité de survie d'un individu de la génération $g$ à la date $t$. Elle est uniquement différentiée selon le genre et la génération sur la base des données des projections de population (Robert-Bobée (2006)). La correction des inégalités d'espérance de vie en fonction de la catégorie socio-professionnelle s'avère beaucoup plus difficile à intégrer (pour une discussion détaillée de ce point, voir Bozio \& Piketty (2008))

- Nous avons supposé que l'introduction du nouveau système s'effectue en 2015 avec une période de transition linéaire de 15 ans. A partir de 2016, chaque nouveau retraité perçoit une pension provenant de l'ancien système (et dépendant donc de son statut socioprofessionnel) et une pension provenant du nouveau système par comptes notionnels (indépendante du statut socioprofessionnel). Au terme de la période de transition (en 2030), le système par comptes notionnels entre pleinement en vigueur et concerne tous les retraités liquidant leur pension. Cependant, toutes les personnes ayant liquidé leur retraite avant 2030 conservent leurs droits acquis dans l'ancien système. Ainsi, même au-delà de 2030, anciens et nouveau systèmes de retraite continuent de coexister, même si la montée en puissance du système par comptes 
notionnels coïncide avec l'extinction progressive des anciennes caisses de retraite au fur et à mesure du vieillissement des retraités les plus âgés. Durant la période de transition (et audelà pour les personnes continuant à toucher des retraites de l'ancien système), les besoins de financement sont générés par l'ancien système. Nous ne supposons aucunes mesures transitoires permettant d'équilibrer les caisses de l'ancien système. En Suède et en Italie, la période de transition était respectivement de 15 ans et de 35 ans.

- Nous supposons l'intégration progressive de tous les régimes actuels (régimes de base et complémentaires des salariés du secteur privé et public et des non salariés) dans un régime unique par comptes notionnels ${ }^{13}$. Dans le système actuel, les plafonds de la sécurité sociale au-delà desquels aucune cotisation n'est prélevée et n'ouvrant pas de droit à pension sont disparates (variant de un à huit fois le plafond de la sécurité sociale en fonction du régime de retraite considéré). Ce choix du niveau "optimal" du plafond détermine l'impact redistributif du système et constitue l'un des paramètres importants de la transition. Nous faisons l'hypothèse simplificatrice d'absence de plafonds dans le cadre de nos simulations.

Les résultats des simulations montrent que, sous l'hypothèse d'économie fermée, l'introduction progressive d'un système par comptes notionnels ainsi paramétré réduit les besoins de financement de l'ordre de 0,6 point de PIB à l'horizon 2050, ce qui représente un cinquième de l'accroissement des besoins de financement de l'ancien système entre 2005 et 2050 (Tableau 3 , scénario A). Cet effet positif est déterminé par une réduction du poids des pensions dans le PIB (-0,7 point de PIB par rapport au modèle de référence) qui se traduit par une réduction progressive du ratio de la pension moyenne au salaire moyen (-20\% entre 2005 et 2050 contre $-18 \%$ dans le modèle de référence), alors que les niveaux de prélèvements restent par hypothèse globalement constants sur l'horizon d'étude.

L'ampleur des effets liés à la mise en place d'un système par comptes notionnels dépend largement du choix des paramètres. Les deux paramètres clés du système sont le taux de rendement des cotisations et le taux d'actualisation des pensions appliqué au moment de la liquidation. Pour assurer la viabilité du système, ces deux paramètres devront être inférieurs ou égaux au taux de croissance de la masse salariale. Mais il existe une forte incertitude sur l'évolution de la productivité du travail et donc du salaire réel. Si l'on retient un taux de rendement des cotisations et un taux d'actualisation des pensions plus faibles et égaux à 1,5\%, $r_{w}(t)=r_{\text {pens }}(t)=1,5 \%$ (alors que le rendement annuel moyen anticipé de la masse salariale entre 2005 et 2050 est d'environ $1,9 \%$ dans le scénario $\mathrm{A}$ ), alors on assiste à une forte réduction des besoins de financement d'un peu plus de deux points et demi de PIB à l'horizon 2050 par rapport au scénario A découlant d'une réduction du ratio de remplacement (Tableau 3, scénario B).

En considérant un scénario extrême où le taux d'actualisation des pensions est nul, $r_{\text {pens }}=0$, (Tableau 3, Scénario C), ce qui revient simplement à déterminer la valeur de la pension en divisant la valeur capitalisée des cotisations individuelles par la durée anticipée de la période de retraite, les pensions servies par le système de retraite se trouvent très nettement réduites

13. Il s'agit ici d'une hypothèse extrême. En pratique, la spécificité des différents régimes pourrait être en partie conservée à l'aide, par exemple, d'un second pilier propre à chaque régime. 
et leur poids dans le PIB diminue d'environ 3,5 points de PIB à l'horizon 2050 par rapport au scénario A. Le système de retraite affiche alors des excédents sur l'ensemble de la période. Les résultats des scénarii $\mathrm{B}$ et $\mathrm{C}$ rappellent également l'importance de raisonner dans un cadre macroéconomique bouclé : la réduction des déficits publics permet une accumulation du capital plus importante, ce qui stimule la croissance économique, réduit le taux de chômage (ce qui a un effet sur la masse des cotisations versées) et réduit le taux d'intérêt (ce qui implique une évolution plus lente de la dette du système de retraite). A l'inverse, le système de comptes notionnels pourrait être paramétré afin de maintenir exactement la générosité du système actuel et, par voie de conséquence, son besoin de financement (ce qui se rapproche du scénario A). En effet, ce n'est pas le système de comptes notionnels qui permet en soit de réduire les besoins de financement mais bien le choix des paramètres du système.

Un objectif possible du système par comptes notionnels pourrait être d'unifier les régimes existants, conformément aux simulations présentées ci-dessus. Une telle unification aurait naturellement un impact différent selon les catégories de travailleurs (Figure 7). En particulier, le passage à un système unique, tel que nous l'avons paramétré, réduit progressivement le taux de remplacement des fonctionnaires ; cette baisse étant d'autant plus marquée que la liquidation de la pension intervient tôt. En effet, l'intégration du régime des fonctionnaires dans un régime commun de droit privé revient à substituer une prise en compte de toutes les années de cotisation à un calcul du salaire de référence actuellement basé sur le dernier salaire. De plus, l'application d'un taux unique de cotisation d'environ $22 \%$ dans le nouveau système ne peut qu' aboutir à une réduction de la générosité du système pour les fonctionnaires étant donné que le taux global de cotisation retraite implicite dépasse actuellement les $60 \%$ du salaire brut dans le secteur public (cotisation salariale de 7,85\% et cotisation patronale implicite de 55,7\% en 2008 en supposant le régime à l'équilibre). Même si de tels ajustements ne sont bien évidemment pas envisageables, ils ont le mérite de souligner la singularité du système actuel de la fonction publique, financé par le budget de l'Etat et donc l'impôt, et donc la difficulté d'une harmonisation entre secteur public et privé.

Pour les cadres, le passage au nouveau système se traduit par un accroissement des taux de remplacement qui s'explique en partie par la suppression du plafond de la sécurité sociale dans nos simulations. Le choix d'un niveau correct du plafond devra alors découler d'un arbitrage entre effets redistributifs (plafonner les retraites les plus hautes) et effets désincitatifs (un plafond trop bas inciterait au développement de retraite sur-complémentaires pour les plus hauts revenus). Enfin, pour les non-cadres, le passage au nouveau système est pénalisant pour ceux qui liquident leur retraite avant 65 ans, mais il est bénéfique dans le cas d'une liquidation plus tardive. De par son aspect contributif, les comptes notionnels conduisent en effet à une meilleure prise en compte des carrières longues et introduisent un mécanisme d'incitation à rester sur le marché du travail beaucoup plus lisible que l'actuel système de décote/surcote, même si cette incitation est évidemment dépendante d'une amélioration du marché de l'emploi des seniors. 
FIGURE 7 - Evolution du ratio " pension moyenne / salaire moyen " (Scénario A, en écart au scénario de référence)

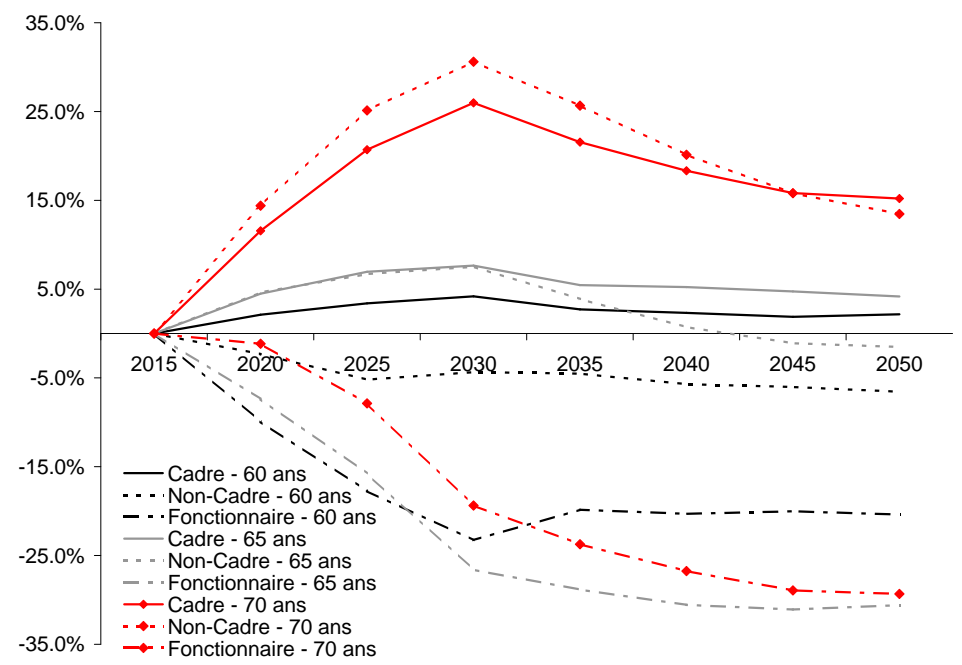

\section{EVALUATION DE LA STABILITÉ DU SYSTÈME PAR COMPTES NOTIONNELS}

La transformation de notre système de retraite actuel en un système par comptes notionnels peut donc avoir des effets positifs en termes de financement. Comme nous l'avons montré, ces effets sont entièrement dépendants du choix des paramètres du nouveau système (en particulier le taux de rendement des cotisations, $r_{w}(t)$, et le taux d'actualisation des pensions, $r_{\text {pens }}$. Toutefois, les gains financiers ne se font qu'au prix d'une réduction de la générosité du système de retraite et donc d'une baisse du niveau relatif des pensions. A côté de cela, le système par comptes notionnels présente également l'avantage de rendre homogène un système qui est actuellement basé sur une multitude de caisses aux règles de fonctionnement différentes, en lui substituant un système dont la gestion est plus simple et transparente. Enfin, les comptes notionnels permettent d'éviter que le pilotage financier du système ne se fasse par un empilement de réformes comme cela est le cas depuis une quinzaine d'années.

Nous allons dans cette section comparer dans quelle mesure un système par comptes notionnels réagit de manière similaire ou différente à un certain nombre de chocs démographiques (variation du taux de fécondité ou de la durée de vie), de chocs économiques (changement du rythme de productivité des facteurs, du taux de chômage ou des taux d'activité) ou de chocs institutionnels (modification du taux de cotisation au système de retraite ou de l'âge légal de départ à la retraite). Pour chacun des chocs considérés, nous étudierons la réaction du système de retraite actuel et de celui par comptes notionnels. Plus précisément, nous focaliseront notre attention sur l'évolution du poids des retraites dans le PIB (Figure 8), sur le besoin de financement (Figure 9) ainsi que sur le ratio entre la pension moyenne et le salaire moyen (Figure 10); l'évolution de chacune des variables étant ici présentée en écart par rapport au scénario de référence s'agissant 
du système de retraite actuel et en écart par rapport au scénario A s'agissant du système par comptes notionnels.

\subsection{Comptes notionnels et chocs démographiques}

L'évolution démographique constitue le principal déterminant de l'évolution d'un système de retraite. Plusieurs travaux récents ont mis l'accent sur la forte incertitude concernant l'évolution démographique future et ses conséquences pour le financement des dépenses de retraites (Plane (2007) et Alho \& Borgy (2007)). En 2007, l'Insee a actualisé ses projections démographiques (voir section 2 pour une présentation plus détaillée de cette actualisation) ce qui conduit à une évolution démographique plus favorable. En 2050, la population active est supérieure de $11,6 \%$ aux anciennes projections et la part des dépenses de retraite dans le PIB (et donc le besoin de financement) est diminué de l'ordre de 1,5 à 2 points de PIB à l'horizon 2050 (Conseil d'Orientation des Retraites (2007) et Chojnicki \& Magnani (2008)). L'un des avantages d'un système par comptes notionnels, système à cotisations définies, tient certainement à sa capacité à s'adapter au moins partiellement aux évolutions démographiques, contrairement au système actuel à prestations définies. Pour s'en rendre compte, nous proposons ici deux variantes d'évolution démographique. Dans la première, nous retenons l'hypothèse haute d'espérance de vie de Robert-Bobée (2006) qui retient des gains plus élevés d'espérance de vie, surtout après 80 ans. Les écarts d'espérance de vie à la naissance par rapport à l'hypothèse centrale sont alors de 2,5 ans en 2050 : 91,5 ans pour les femmes et 86,3 ans pour les hommes selon l'hypothèse haute contre respectivement 89 ans et 83,8 ans dans le scénario central. Dans la seconde variante, nous retenons le scénario de fécondité basse de Robert-Bobée (2006) qui suppose une baisse progressive de celle-ci jusqu'à 1,7 enfant par femme en 2010 (contre 1,9 dans le scénario central) puis son maintien ; ce seuil correspondant à la fécondité minimale des cinquante dernières années.

Le tableau 4 présente l'impact sur l'évolution de la population de chacune de ces deux variantes démographiques. Dans l'hypothèse d'espérance de vie haute, la population totale croît d'un peu plus de $2 \%$ à l'horizon 2050 par rapport au scénario démographique de référence mais c'est naturellement la population des plus de 65 ans qui est affectée $(+8,3 \%)$ alors que la population active demeure globalement stable. Le ratio de dépendance augmente alors de près de 4 points de pourcentage et le nombre de cotisants par retraité diminue de 7\%. Dans le système de retraite actuel, une telle perspective démographique se traduit naturellement par un accroissement marqué de la part des retraites dans le PIB (Figure 8) et in fine du besoin de financement (Figure 9) alors que dans le système par comptes notionnels, les tensions à la hausse sont nettement contenues. En effet, le système par comptes notionnels tient compte dans le calcul des retraites des gains d'espérance de vie et répercute progressivement ceux-ci dans le calcul des pensions liquidées. Ainsi, le niveau relatif des pensions diminue nettement dans le système de retraite par comptes notionnels alors qu'il demeure globalement stable dans le système actuel (Figure 10).

Si l'on considère à présent une dégradation du ratio de dépendance découlant de l'hypothèse 
basse de fécondité, la population totale décroît nettement à l'horizon $2050(-5,8 \%)$ du fait de la baisse de la population en âge d'activité (Tableau 4). La population des retraités n'est pas affectée ici du fait de la proximité de l'horizon de projection. Dans cette hypothèse de "vieillissement par le bas" plus marqué de la population française, les deux systèmes de retraite considérés réagissent exactement de la même manière avec un accroissement de la part des retraites dans le PIB (du fait d'une moindre croissance de celui-ci), une dégradation du besoin de financement (la baisse de la population active se traduisant par une baisse de la masse des cotisations) et une dégradation à partir de 2020 du ratio de la pension moyenne au salaire moyen (le facteur travail devenant plus rare, sa productivité augmente). En effet, le système par comptes notionnels demeure un système par répartition : les actifs financent les pensions des retraités de la période et toute modification du nombre de cotisants pour un nombre donné de retraités modifie instantanément les conditions d'équilibre du système.

TABLEAU 4 - Perspectives d'évolution de la population française selon deux scénarii alternatifs

\begin{tabular}{|c|c|c|c|c|c|c|}
\hline & 2005 & 2010 & 2020 & 2030 & 2040 & 2050 \\
\hline & \multicolumn{6}{|c|}{ Variante espérance de vie Haute } \\
\hline Population totale (en milliers) & 60702 & 62343 & 65232 & 67733 & 69960 & 71556 \\
\hline Population en âge de travailler (15-64 ans, en milliers) & 39531 & 40537 & 40521 & 40372 & 40037 & 40287 \\
\hline Population de plus de 65 ans & 9968 & 10411 & 13303 & 16105 & 18540 & 19849 \\
\hline Ratio de dépendance (Pop 65 et plus / Pop 15-64 en \%) & $25,2 \%$ & $25,7 \%$ & $32,8 \%$ & $39,9 \%$ & $46,3 \%$ & $49,3 \%$ \\
\hline Nombre de cotisants (en milliers) & 25096 & 25460 & 26144 & 26123 & 26246 & 26167 \\
\hline Nombre de retraités (en milliers) & 12592 & 13880 & 16794 & 19495 & 21458 & 22911 \\
\hline \multirow[t]{2}{*}{ Nombre de cotisants par retraité } & 1,99 & 1,83 & 1,56 & 1,34 & 1,22 & 1,14 \\
\hline & \multicolumn{6}{|c|}{ Variante Fécondité basse } \\
\hline Population totale (en milliers) & 60702 & 62143 & 64040 & 65474 & 66290 & 65886 \\
\hline Population en âge de travailler (15-64 ans, en milliers) & 39531 & 40532 & 40499 & 39780 & 38656 & 38050 \\
\hline Population de plus de 65 ans & 9968 & 10377 & 13080 & 15620 & 17656 & 18323 \\
\hline Ratio de dépendance (Pop 65 et plus / Pop 15-64 en \%) & $25,2 \%$ & $25,6 \%$ & $32,3 \%$ & $39,3 \%$ & $45,7 \%$ & $48,2 \%$ \\
\hline Nombre de cotisants (en milliers) & 25096 & 25457 & 26129 & 25978 & 25597 & 24958 \\
\hline Nombre de retraités (en milliers) & 12592 & 13845 & 16568 & 19003 & 20565 & 21372 \\
\hline Nombre de cotisants par retraité & 1,99 & 1,84 & 1,58 & 1,37 & 1,24 & 1,17 \\
\hline
\end{tabular}

Insee (2006), Calculs des auteurs

\subsection{Comptes notionnels et chocs économiques}

Les conditions d'équilibre d'un système de retraite sont ensuite déterminées par les évolutions économiques et en particulier par l'évolution de la productivité globale des facteurs (PGF), l'évolution du chômage et des taux d'activité. A nouveau, nous comparons la réaction de chacun des deux systèmes de retraite au regard de l'évolution de ces trois paramètres. S'agissant de la PGF, nous considérons un scénario pessimiste d'évolution où celle-ci croît à un rythme plus faible de 1,5\% par an à partir de 2010, ce qui correspond au taux de croissance de la productivité observé sur la période 2001 - 2007 (Conseil d'Orientation des Retraites (2010a)). Une 
moindre croissance de la PGF se traduit automatiquement par un rythme de croissance du PIB moins soutenu et ce quel que soit les modalités de fonctionnement du système de retraite (de l'ordre de $-0,35$ à $-0,5$ point de croissance en 2050 selon le système). Pour autant, les effets sont nettement plus marqués dans le cas du système de retraite actuel : la part des dépenses dans le PIB augmente de près de 1,5 point de PIB contre moins de 0,5 point de PIB dans le système par comptes notionnels. En effet, la baisse du rythme de croissance du PIB se traduit dans les deux cas par une baisse de la masse des cotisations mais la masse des pensions versées diminue dans le système par comptes notionnels du fait de la prise en compte de la baisse du rythme de la productivité dans le taux de rendement appliqué aux cotisations passées, $r_{w}(t)$ et dans le taux d'actualisation des pensions au moment de la liquidation $r_{p e n s}(\mathrm{t})$. A l'inverse, la masse des pensions en valeur demeure globalement stable dans le système actuel du fait de l'indexation des salaires servant au calcul des retraites ainsi que des pensions sur l'inflation.

Nous considérons ensuite un scénario d'évolution pessimiste du taux de chômage sur l'horizon de projection. En particulier, nous faisons l'hypothèse que celui-ci demeure constant à partir de 2010 à 9,5\% alors que dans le scénario de référence le taux de chômage décroît progressivement vers une cible de $7 \%$ en 2020. Les effets de ce scénario pessimiste d'évolution du marché du travail sont quasiment symétriques mais d'une ampleur légèrement plus faible dans le cas des comptes notionnels : le rythme de croissance du PIB diminue et le niveau relatif des dépenses de retraite augmente donc immédiatement (Figure 8); la réduction du nombre d'actifs occupés (et donc de cotisants) accroît les besoins de financement du système de retraite (Figure 9); la raréfaction du facteur travail accroît le rendement du travail et donc réduit le ratio pension moyenne/salaire moyen jusque 2020, la tendance s'inversant ensuite progressivement (et plus fortement dans le système de retraite actuel) du fait d'une réduction du niveau des pensions découlant de l'intégration des périodes de chômage dans le calcul des pensions (Figure 10).

Ces mécanismes peuvent être en grande partie transposés dans le cas d'une réduction des taux d'activité qui constituent le dernier paramètre clé de l'évolution économique sur le fonctionnement du système de retraite. En particulier, nous supposons ici une constance des comportements d'activité entre 2010 et 2050 alors que notre scénario d'activité de référence est basé sur les projections de l'Insee qui prévoient notamment que les taux d'activité des séniors progressent de tel sorte que le taux d'emploi des 55-59 ans passe de 60\% en 2005 à 63\% en 2050 et celui des 60-64 ans de 20\% à 40\% (Figure 3). Dans les deux systèmes de retraite, les implications de taux d'activité plus faibles sont importantes à court et à moyen terme : le nombre de retraités bénéficiant d'une pension plus faible reste limité ; la baisse des taux d'activité réduisant en parallèle le nombre de cotisants et accroissant par conséquent le besoin de financement. A long terme en revanche, le nombre de retraités qui perçoivent une pension plus faible progresse, beaucoup plus dans le système par comptes notionnels qui est entièrement contributif que dans le système actuel qui répercute moins les accidents de carrière. 


\subsection{Comptes notionnels et chocs institutionnels}

La fixation des paramètres institutionnels du système de retraite détermine en grande partie son mode de fonctionnement et par là même son évolution en termes de besoins de financement. En particulier, le taux de cotisation joue un rôle totalement différent dans l'équilibre du système de retraite actuel et dans un système par comptes notionnels. Pour bien le percevoir, nous augmentons les taux de cotisation du système de retraite de $10 \%$ à partir de 2010 ( $\tau$ (" $r b$ ") dans le régime de base, $\tau_{1}(c)$ et $\tau_{2}(c)$ dans le régime complémentaire et $\tau(" r f$ ") dans le régime de la fonction publique) et le taux de cotisation unique du système par compte notionnel $\left(\tau_{c n}\right)$ à partir de la mise en place de celui-ci en 2015. Cet accroissement du taux de cotisation se traduit immédiatement et mécaniquement par un accroissement de la masse des prélèvements des régimes de retraite actuel ce qui réduit le besoin de financement du système, alors que cet effet positif n'est que très temporaire dans le cas du système par comptes notionnels (Figure 9). En effet, dans le nouveau système, tout accroissement du taux de cotisation se répercute à terme par une augmentation des pensions versées puisque le système est à cotisations définies ; le ratio de la pension moyenne au salaire moyen augmente alors progressivement sur l'horizon d'étude (Figure 10) et le poids des pensions dans le PIB suit la même tendance (Figure 8). A l'inverse, dans le système actuel, la masse des pensions versées demeure constante et le poids relatifs des dépenses de retraite tend à diminuer. En effet, la réduction des besoins de financement desserre en partie la contrainte financière de l'Etat et permet une réaffectation de l'épargne publique vers de l'investissement productif qui stimule la croissance et permet un rythme de croissance plus soutenu. Ainsi, si le choix du taux de cotisation constitue l'un des trois leviers d'action (avec le niveau des pensions et l'âge effectif de départ à la retraite) de l'équilibre du système de retraite actuel, il disparaît dans le cadre du système par comptes notionnels pour devenir un simple élément de générosité de celui-ci.

Pour terminer, le choix de l'âge légal de départ à la retraite est souvent présenté comme l'un des paramètres déterminants de l'équilibre financier d'un système de retraite. Jusqu'à présent, toutes nos simulations ont maintenu ce paramètre constant et égal à 60 ans. Nous supposons maintenant une augmentation progressive de celui-ci à 62 ans d'ici à 2020 couplé à une hypothèse très optimiste de décalage d'une ampleur équivalente des taux d'activité des individus âgés de 60 à 62 ans ${ }^{14}$. En effet, de nombreuses études ont présenté cette précocité de l'âge légal de départ à la retraite (la France est l'un des pays des l'OCDE où cet âge est le plus faible) comme l'une des explications de la faiblesse des taux d'activité des séniors (d'Autume, Betbèze \& Hairault (2006) et Hairault, Langot \& Sopraseuth (2008)). Une telle réforme aboutit à des effets très similaires dans les deux systèmes envisagés. A court terme, l'augmentation des taux d'activité des séniors (et le décalage de la liquidation) permet de réduire les dépenses de retraite et par conséquent les besoins de financement. Par contre, à long terme, le nombre de retraités qui perçoivent une pension plus élevée progresse, ce qui neutralise l'effet positif produit par l'augmentation du volume des cotisations versées. Il convient de noter que les effets

14. Sans décalage des taux d'activité, l'augmentation de l'âge légal de départ à la retraite se traduit dans les deux systèmes par une réduction des taux de remplacement. 
sont très proches dans les deux systèmes puisque les coefficients de décote et de surcote du système actuel sont très proches de la neutralité actuarielle découlant d'un système par comptes notionnels.

\section{Conclusion}

Cet article évalue les effets d'une transformation du système actuel de retraite français en un système par comptes notionnels à l'aide d'un modèle d'équilibre général à générations imbriquées. Contrairement à la plupart des modèles utilisés dans ce type d'analyse, cette modélisation permet de prendre en compte les effets de bouclage macroéconomique pour différents environnements d'ouverture économique. Ainsi, les effets de retour des réformes sur les marchés du capital et du travail ainsi que sur la croissance sont calculés par le modèle et non définis de manière exogène, ce qui affecte finalement l'effet des réformes sur l'équilibre des régimes des retraites.

En particulier, nous simulons les effets de la mise en place progressive, entre 2015 et 2030, d'un système unique par comptes notionnels se substituant entièrement, à terme, à tous les régimes actuels, de base et complémentaires. L'introduction d'un système de comptes notionnels nécessite plusieurs choix de la part du décideur politique. En particulier, nous considérons ici un taux de cotisation qui est fixé de manière à maintenir constante la masse des cotisations découlant de l'ancien système à la date de début de la transition, un taux de rendement appliqué aux cotisations versées égal au taux de croissance annuel du PIB, un taux d'actualisation des pensions égal au taux de croissance de la productivité et une indexation des pensions sur les prix. Par rapport à notre scénario de référence, les résultats des simulations montrent que l'introduction progressive d'un système par comptes notionnels permettrait d'obtenir une importante réduction des besoins de financement de l'ordre de 0,6 point de PIB à l'horizon 2050.

Nous avons ensuite évalué la stabilité du système par comptes notionnels, en comparant dans quelle mesure ce nouveau système réagit à un certain nombre de chocs démographiques, de chocs économiques ou de chocs institutionnels. Pour chacun des chocs considérés, nous étudions la réaction des deux systèmes de retraite (le système de retraite actuel et celui par comptes notionnels). En considérant les effets sur les besoins de financement, les résultats de nos simulations montrent que le système par comptes notionnels réagit mieux, par rapport au système actuel, dans le cas d'une augmentation de l'espérance de vie, d'une baisse du taux de croissance de la productivité et d'une baisse des taux d'activité. Le nouveau système réagit de façon très similaire dans le cas d'une baisse de la fécondité et d'une hausse du taux de chômage. Par contre, dans le cas d'une hausse des cotisations, l'effet sur les besoins de financement dans un système par compte notionnels est évidemment négatif, du fait d'un accroissement parallèle de la valeur des pensions. Ainsi, face aux nombreuses incertitudes économiques et démographiques, un système par comptes notionnels présente l'avantage d'une meilleure adaptabilité aux évolutions observées. Plus qu'une formule miracle, c'est cette propriété dynamique qui peut être recherchée dans la réforme structurelle des régimes de retraite. 


\section{Figure 8 - Evolution des dépenses de retraite (en \% du PIB)}

(a) Système de retraite actuel - Ecart en points de \% par rapport au scénario de référence

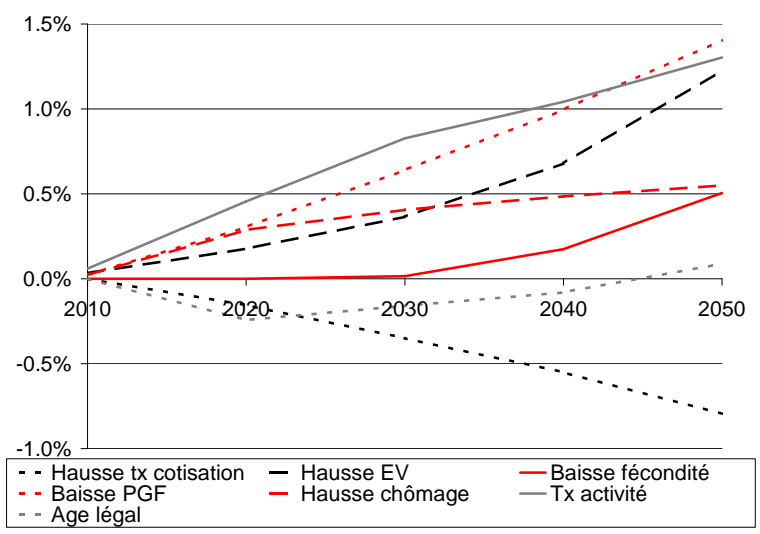

(b) Système de retraite par comptes notionnels - Ecart en points de \% par rapport au scénario $\mathrm{A}$

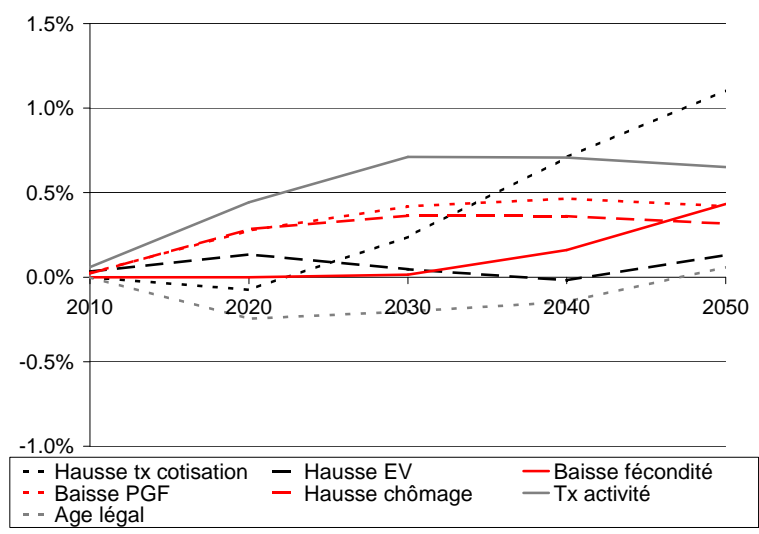

FIgURE 9 - Evolution du besoin de financement

(a) Système de retraite actuel - Ecart en points de \% par rapport au scénario de référence

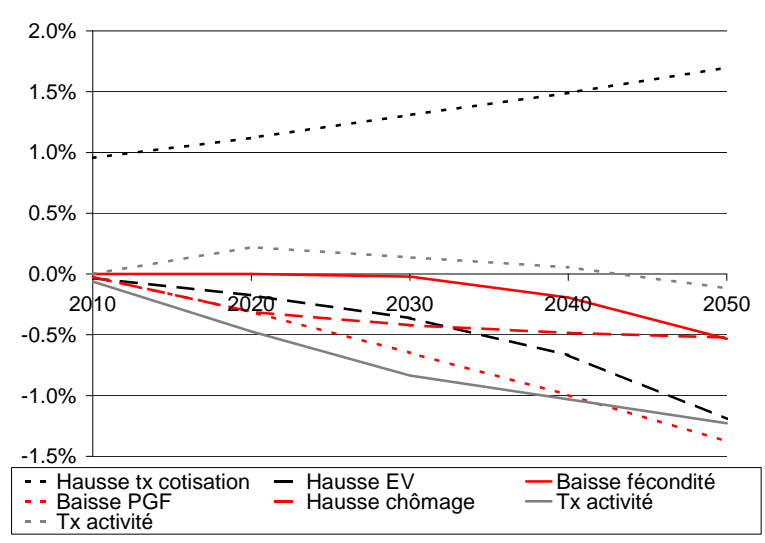

(b) Système de retraite par comptes notionnels - Ecart en points de \% par rapport au scénario A

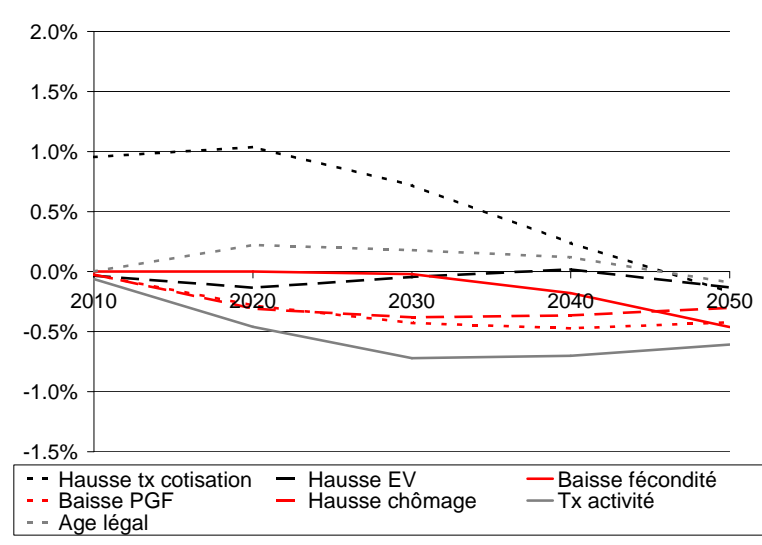

FigURE 10 - Evolution du ratio pension moyenne/salaire moyen

(a) Système de retraite actuel - Ecart en points de \% par rapport au scénario de référence

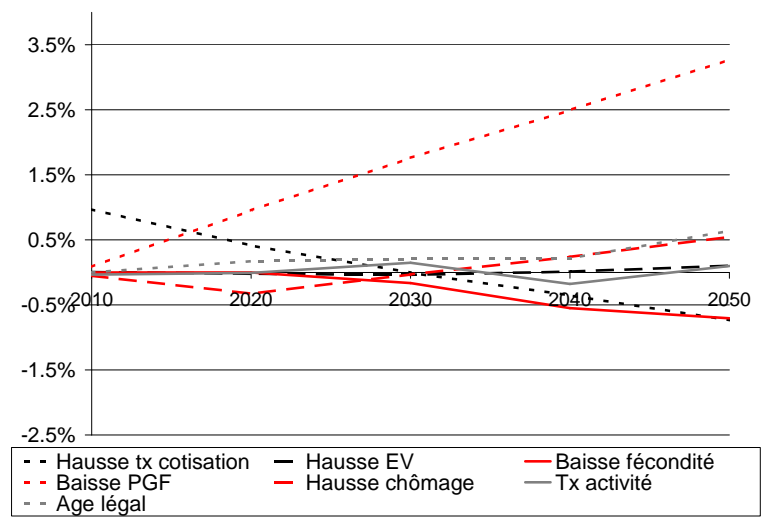

(b) Système de retraite par comptes notionnels - Ecart en points de \% par rapport au scénario $\mathrm{A}$

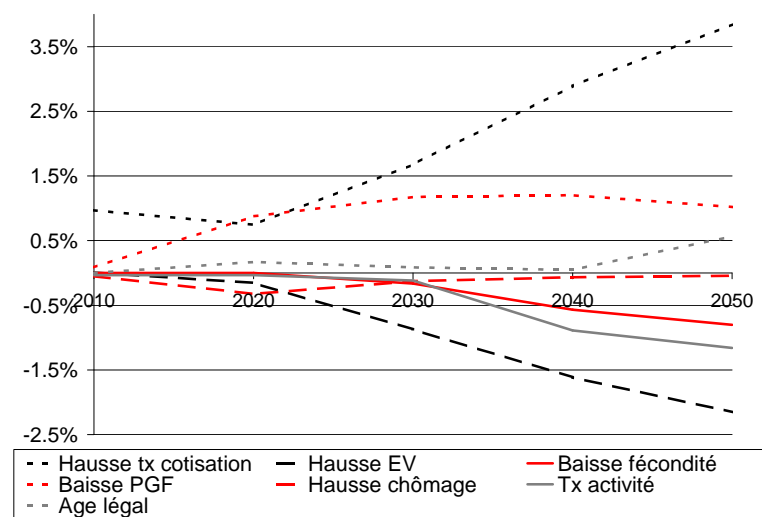




\section{RÉFÉRENCES}

Alho, J. \& Borgy, V. (2007), 'Global ageing and macroeconomic consequences of demographic uncertainty in a multi-regional model', CEPII-Document de travail 2007-09.

Arrondel, L. \& Laferrère, A. (2001), 'Taxation and wealth transmission in france', Journal of Public Economics 79.

Auerbach, A. \& Kotlikoff, L. (1987), Dynamic Fiscal Policy, Cambridge University Press.

Bac, C., Bonnet, C., Bontout, O. \& Cornilleau, G. (2003), 'Exercices de simulation de l'évolution à long terme du système de retraite', Document de travail, Série Etude 38.

Bardaji, J., Sédillot, B. \& Walraet, E. (2004), 'Les retraites du secteur public : projections à l'horizon 2040 à l'aide du modèle de microsimulation destinie', Document de Travail de l'Insee G2004/14.

Blanchet, D. (1992), 'Retraites et croissance à long terme : un essai de simulation', Economie et Prévision 105.

Bozio, A. \& Piketty, T. (2008), 'Pour un nouveau système de retraite - des comptes individuels de cotisation financée par répartition', Opuscule du Cepremap .

Brender, A. \& Gabrielli, D. (2001), 'Les aspects financiers du vieillissement de la population', Bulletin de la Banque de France 92.

Börsch-Supan, A., Ludwig, A. \& Winter, A. (2006), 'Aging, pension reform and capital flows', Economica 73.

Brutel, C. \& Omalek, L. (2003), 'Projections pour la france, ses régions et ses départements (horizon 2030/2050)', Insee Résultats 16.

Chateau, J., Chojnicki, X. \& Magnani, R. (2009), 'Disparities in pension systems and financial flows among european countries', Journal of Pension Economics and Finance 8-1.

Chojnicki, X. \& Magnani, R. (2008), 'Vieillissement, retraite et ouverture financière en europe : des réformes encore insuffisantes', Economie Internationale 113.

Conseil d'Orientation des Retraites (2001), 'Retraites : renouveler le contrat social entre les générations', Premier rapport au Premier Ministre, COR .

Conseil d'Orientation des Retraites (2006), 'Retraites : Perspectives 2020 et 2050', Troisième rapport au Premier Ministre, COR .

Conseil d'Orientation des Retraites (2007), 'Retraites : 20 fiches d'actualisation pour le rendez-vous de 2008', Cinquième rapport au Premier Ministre, COR .

Conseil d'Orientation des Retraites (2010a), 'Retraites : Perspectives actualisées à moyen et long terme en vue du rendez-vous de 2010', Huitième rapport au Premier Ministre, COR . 
Conseil d'Orientation des Retraites (2010b), 'Retraites : annuités, points ou comptes notionnels ? options et modalités techniques', Septième rapport au Premier Ministre .

d'Autume, A., Betbèze, J. \& Hairault, J. (2006), Les seniors et l'emploi en France, Conseil d'Analyse Economique.

d'Autume, A. \& Quinet, A. (2001), 'Une maquette de moyen terme de l'économie française', Economie et Prévision 148.

Diamond, P. (1965), 'National debt in a neoclassical growth model', American Economic Review 55.

Hairault, J., Langot, F. \& Sopraseuth, T. (2004), 'Retraite choisie et réduction des déficits', Revue française d'économie 18.

Hairault, J., Langot, F. \& Sopraseuth, T. (2008), 'Pour une retraite choisie - l'emploi des seniors', Opuscule du Cepremap.

Hénin, P. \& Weitzenblum, T. (2004), 'Eléments d'évaluation de la réformes des retraites', Revue française d'économie 18.

Hourriez, J. \& Olier, L. (1997), 'Niveau de vie et taille du ménage : estimation d'une échelle d'équivalence', Economie et Statistique 308-309-310.

Imrohoroglu, S. (1998), 'A quantitative analysis of capital income taxation', International Economic Review 39-2.

INGENUE (2006), 'The larger europe : technological convergence and the labour migration', Revue Economique 57-4.

Marchand, S., Massal, P. \& Raynaud, P. (2002), 'Situation et perspectives démographiques de la fonction publique de l'etat', Mimeo du bureau des statistiques, des études et de l'évaluation, DGAFP.

Plane, M. (2007), 'La démographie à la rescousse de la protection sociale en france', La Lettre de l'OFCE 281.

Robert-Bobée, I. (2006), 'Projections de population 2005-2050 pour la france métropolitaine : méthode et résultats', Document de travail de l'Insee $\mathbf{F 0 6 0 3 . ~}$

Samuelson, P. (1958), 'An exact consumption-loan model of interest with or without the social contrivance of money', Journal of Political Economy 66-6. 


\section{LISTE DES DOCUMENTS DE TRAVAIL REALISES PAR LE CEPII}

Une liste complète est disponible sur le site du CEPII : |lwww.cepii.fr. Pour recevoir un message d'alerte, contacter Sylvie Hurion (sylvie.hurion@cepii.fr).

$N^{\circ}$

2010-18 Clustering the Winners: The French Policy of Competitiveness Clusters

2010-17 The Credit Default Swap Market and the Settlement of Large Defauts

2010-16 The Impact of the 2007-10 Crisis on the Geography of Finance

2010-15 Socially Responsible Investing: it takes more than Words

2010-14 A Case for Intermediate Exchange-Rate Regimes

2010-13 Gold and Financial Assets: Are There Any Safe Havens in Bear Markets?

2010-12 European Export Performance

2010-11 The Effects of the Subprime Crisis on the Latin American Financial Markets: An Empirical Assessment

2010-10 Foreign Bank Presence and its Effect on Firm Entry and Exit in Transition Economies

2010-09 The Distorted Effect of Financial Development on International Trade Flows

2010-08 Exchange Rate Flexibility across Financial Crises
L. Fontagné, P. Koenig, F. Mayneris \& S. Poncet

V. Coudert \& M. Gex

G. Capelle-Blancard \& Y. Tadjeddine

G. Capelle-Blancard \& S. Monjon

V. Salins \& A. Bénassy-Quéré V. Coudert \& H. Raymond

A. Cheptea, L. Fontagné \& S. Zignago

G. Dufrénot, V. Mignon \& A. Péguin-Feissolle

O. Havrylchyk

A . Berthou

V. Coudert, C. Couharde \& V. Mignon 
$N^{\circ}$

2010-07 Crises and the Collapse of World Trade: the Shift to Lower Quality

2010-06 The heterogeneous effect of international outsourcing on firm productivity

2010-05 Fiscal Expectations on the Stability and Growth Pact: Evidence from Survey Data

2010-04 Terrorism Networks and Trade: Does the Neighbor Hurt

2010-03 Wage Bargaining and the Boundaries of the Multinational Firm

2010-02 Estimation of Consistent Multi-Country FEERs

2010-01 The Elusive Impact of Investing Abroad for Japanese Parent Firms: Can Disaggregation According to FDI Motives Help

2009-39 The Effects at Home of Initiating Production Abroad: Evidence from Matched French Firms

2009-38 On Equilibrium Exchange Rates: Is Emerging Asia Different?

2009-37 Assessing Barriers to Trade in the Distribution and Telecom Sectors in Emerging Countries

2009-36 Les impacts économiques du changement climatique : enjeux de modélisation

2009-35 Trade, Foreign Inputs and Firms’ Decisions: Theory and Evidence

2009-34 Export Sophistication and Economic Performance: Evidence from Chinese Provinces

2009-33 Assessing the Sustainability of Credit Growth: The Case of Central and Eastern European Countries

2009-32 How do different exporters react to exchange rate changes? Theory, empirics and aggregate implications

2009-31 Spillovers from Multinationals to Heterogeneous Domestic Firms: Evidence from Hungary

2009-30 Ethnic Networks, Information, and International Trade: Revisiting the Evidence

2009-29 Financial Constraints in China: Firm-level Evidence

\section{Auteurs}

A. Berthou \& C. Emlinger

F. McCann

M. Poplawski-Ribeiro \& J.C. Rüle

J. de Sousa, D. Mirza \& T. Verdier

M. Bas \& J. Carluccio

B. Carton \& K. Hervé

L. Hering, T. Inui \& S. Poncet

A. Hijzen, S. Jean \& T. Mayer

A. López-Villavicencio \& V. Mignon

L. Fontagné \& C. Mitaritonna

P. Besson \& N. Kousnetzoff

M. Bas

J. Jarreau \& S. Poncet

V. Coudert \& C. Pouvelle

N. Berman, P. Martin \& Thierry Mayer

G. Békés, J. Kleinert \& F. Toubal

G. J. Felbermayr, B. Jung \& F. Toubal

S. Poncet, W. Steingress \& H. Vandenbussche 
$N^{\circ}$

2009-28 The Crisis: Policy Lessons and Policy Challenges

2009-27 Commerce et flux financiers internationaux : MIRAGE-D

2009-26 Oil Prices, Geography and Endogenous Regionalism: Too Much Ado about (Almost) Nothing

2009-25 EU15 Trade with Emerging Economies and Rentier States: Leveraging Geography

2009-24 Market Potential and Development

2009-23 Immigration, Income and Productivity of Host Countries: A Channel Accounting Approach

2009-22 A Picture of Tariff Protection Across the World in 2004 MAcMap-HS6, Version 2

2009-21 Spatial Price Discrimination in International Markets

2009-20 Is Russia Sick with the Dutch Disease

2009-19 Économies d'agglomération à l'exportation et difficulté d'accès aux marchés

2009-18 Local Export Spillovers in France

2009-17 Currency Misalignments and Growth: A New Look using Nonlinear Panel Data Methods,

2009-16 Trade Impact of European Measures on GMOs Condemned by the WTO Panel

2009-15 Economic Crisis and Global Supply Chains

2009-14 Quality Sorting and Trade: Firm-level Evidence for French Wine

2009-13 New Evidence on the Effectiveness of Europe's Fiscal Restrictions

2009-12 Remittances, Capital Flows and Financial Development during the Mass Migration Period, 1870-1913

2009-11 Evolution of EU and its Member States'Competitiveness in International Trade

\section{Auteurs}

A. Bénassy-Quéré, B. Coeuré, P. Jacquet \&J. Pisani-Ferry

A. Lemelin

D. Mirza \& H. Zitouna

G. Gaulier, F. Lemoine \& D. Ünal

T. Mayer

A. Mariya \& A. Tritah

H. Boumellassa, D. Laborde Debucquet \& C. Mitaritonna

J. Martin

V. Dobrynskaya \& E. Turkisch

P. Koenig, F. Mayneris \& S. Poncet

P. Koenig, F. Mayneris \& S. Poncet

S. Béreau,

A. López Villavicencio \& V. Mignon

A. C. Disdier \& L. Fontagné

A. Bénassy-Quéré, Y. Decreux, L. Fontagné \& D. Khoudour-Casteras

M. Crozet, K. Head \& T. Mayer

M. Poplawski Ribeiro

R. Esteves \& D. Khoudour-Castéras

L. Curran \& S. Zignago 
$N^{\circ}$

2009-10 Exchange-Rate Misalignments in Duopoly: The Case of Airbus and Boeing

2009-09 Market Positioning of Varieties in World Trade: Is Latin America Losing out on Asia?

2009-08 The Dollar in the Turmoil

2009-07 Term of Trade Shocks in a Monetary Union: An Application to West-Africa

2009-06 Macroeconomic Consequences of Global Endogenous Migration: A General Equilibrium Analysis

2009-05 Équivalence entre taxation et permis d’émission échangeables

2009-04 The Trade-Growth Nexus in the Developing Countries: a Quantile Regression Approach

2009-03 Price Convergence in the European Union: within Firms or Composition of Firms?

2009-02 Productivité du travail : les divergences entre pays développés sont-elles durables?

2009-01 From Various Degrees of Trade to Various Degrees of Financial Integration: What Do Interest Rates Have to Say

\section{Auteurs}

A. Bénassy-Quéré, L. Fontagné \& H. Raff

N. Mulder, R. Paillacar \& S. Zignago

A Bénassy-Quéré, S. Béreau \& V. Mignon

L. Batté, A. Bénassy-Quéré, B. Carton \& G. Dufrénot V. Borgy, X. Chojnicki, G. Le Garrec \& C. Schwellnus

$$
\text { P. Villa }
$$

G. Dufrénot, V. Mignon \& C. Tsangarides

I. Méjean \& C. Schwellnus

C. Bosquet \& M. Fouquin

A. Bachellerie, J. Héricourt \& V. Mignon 
Organisme public d'étude et de recherche en économie internationale, le CEPII est placé auprès du Centre d'Analyse Stratégique. Son programme de travail est fixé par un conseil composé de responsables de l'administration et de personnalités issues des entreprises, des organisations syndicales et de l’Université.

Les documents de travail du CEPII mettent à disposition du public professionnel des travaux effectués au CEPII, dans leur phase d'élaboration et de discussion avant publication définitive. Les documents de travail sont publiés sous la responsabilité de la direction du CEPII et n'engagent ni le conseil du Centre, ni le Centre d'Analyse Stratégique. Les opinions qui y sont exprimées sont celles des auteurs.

Les documents de travail du CEPII sont disponibles sur le site : http//www.cepii.fr.

\section{CEPII}

9, RUe Georges Pitard, 75740 Paris CedeX 15

SYLVIE HURION - PUBLICATIONS

TÉL : 0153685514 - FAX : 0153685504

sylvie.hurion@cepii.fr

ISSN : 1293-2574 